\title{
Spin-charge coupling effects in a two-dimensional electron gas
}

\author{
Roberto Raimondi \\ Dipartimento di Matematica e Fisica, Università Roma Tre, \\ Via della Vasca Navale 84, Rome, 00146, Italy \\ E-mail: roberto.raimondi@uniroma3.it \\ www.uniroma3.it \\ Cosimo Gorini \\ Institut für Theoretische Physik, Universität Regensburg, \\ 93040 Regensburg, Germany \\ E-mail: cosimo.gorini@physik.uni-regensburg.de \\ Sebastian Tölle \\ Institut für Physik, Universität Augsburg \\ Universitätsstr. 1, 86135 Augsburg, Germany \\ E-mail: sebastian.toelle@physik.uni-augsburg.de
}

\begin{abstract}
In these lecture notes we study the disordered two-dimensional electron gas in the presence of Rashba spin-orbit coupling, by using the Keldysh nonequilibrium Green function technique. We describe the effects of the spin-orbit coupling in terms of a $\mathrm{SU}(2)$ gauge field and derive a generalized Boltzmann equation for the charge and spin distribution functions. We then apply the formalism to discuss the spin Hall and the inverse spin galvanic (Edelstein) effects. Successively we show how to include, within the generalized Boltzmann equation, the side jump, the skew scattering and the spin current swapping processes originating from the extrinsic spin-orbit coupling due to impurity scattering.
\end{abstract}

Keywords: Spin-orbit coupling; Electronic transport; Many-body Green function; Disordered systems.

\section{Introduction}

These lecture notes are based mainly on the work by Gorini et al. of Ref. 1, where by means of a gradient expansion a generalized Boltzmann equation with SU(2) gauge fields was obtained for the disordered Rashba model. The inclusion of the extrinsic spin-orbit coupling (SOC) from impurities in the $\mathrm{SU}(2)$ formalism was later considered in the work by Raimondi et al. in Ref. 2. Hence, the aim of these lecture notes is to provide a self-contained and 
pedagogical introduction to the disordered two-dimensional electron gas (2DEG) with both intrinsic (Rashba) and extrinsic SOC within the SU(2) gauge-field approach. The lecture notes by Tatara in this series are a good complementary reading dealing with electron transport in ferromagnetic metals ${ }^{3}$.

The layout of these lecture notes is the following. In Section 2 we write down the quantum kinetic equation for the fermion Green function in the presence of $\mathrm{U}(1)$, associated with the electromagnetic field, and $\mathrm{SU}(2)$ gauge fields. The standard model of disorder is introduced in Section 3. Whereas in Section 2 we derive the hydrodynamic SU(2) derivative of the Boltzmann equation, in Section 3 we obtain an expression for the collision integral describing the scattering from impurities. In Section 4 we apply the formalism to the disordered Rashba model and derive the Bloch equation for the spin density, describe the Dyakonov-Perel spin relaxation, and discuss a thermally induced spin polarization. In Section 5 we introduce SOC from impurity scattering and analyze the so-called side jump mechanism, which manifests as a correction to the velocity operator. In Section 6 we discuss the skew scattering mechanism. Both intrinsic and extrinsic SOCs contribute to the spin relaxation. Extrinsic SOC gives rise to Elliott-Yafet spin relaxation, which is covered in Section 7, together with the complete form of the Boltzmann equation. Section 8 states our conclusions. Throughout we use units such that $\hbar=c=1$.

\section{The kinetic equation and the $\mathrm{SU}(2)$ covariant Green function}

We begin by defining the Keldysh Green function (for an introduction see, e.g., the book by Rammer ${ }^{4}$ )

$$
\check{G}=\left(\begin{array}{cc}
G^{R} & G^{K} \\
0 & G^{A}
\end{array}\right),
$$

where the retarded $G^{R}$, Keldysh $G^{K}$ and advanced $G^{A}$ components are given by

$$
\begin{aligned}
& G^{R}\left(\mathbf{r}_{1}, t_{1} ; \mathbf{r}_{2}, t_{2}\right)=-i \Theta\left(t_{1}-t_{2}\right)\left\langle\psi\left(\mathbf{r}_{1}, t_{1}\right) \psi^{\dagger}\left(\mathbf{r}_{2}, t_{2}\right)+\psi^{\dagger}\left(\mathbf{r}_{2}, t_{2}\right) \psi\left(\mathbf{r}_{1}, t_{1}\right)\right\rangle \\
& G^{K}\left(\mathbf{r}_{1}, t_{1} ; \mathbf{r}_{2}, t_{2}\right)=-i\left\langle\psi\left(\mathbf{r}_{1}, t_{1}\right) \psi^{\dagger}\left(\mathbf{r}_{2}, t_{2}\right)-\psi^{\dagger}\left(\mathbf{r}_{2}, t_{2}\right) \psi\left(\mathbf{r}_{1}, t_{1}\right)\right\rangle \\
& G^{A}\left(\mathbf{r}_{1}, t_{1} ; \mathbf{r}_{2}, t_{2}\right)=i \Theta\left(t_{2}-t_{1}\right)\left\langle\psi\left(\mathbf{r}_{1}, t_{1}\right) \psi^{\dagger}\left(\mathbf{r}_{2}, t_{2}\right)+\psi^{\dagger}\left(\mathbf{r}_{2}, t_{2}\right) \psi\left(\mathbf{r}_{1}, t_{1}\right)\right\rangle .
\end{aligned}
$$

In the above definitions $\psi\left(\mathbf{r}_{1}, t_{1}\right)$ and $\psi^{\dagger}\left(\mathbf{r}_{2}, t_{2}\right)$ are Heisenberg field operators for fermions and $\Theta(t)$ the Heaviside step function. In the following we 
will be concerned with spin one-half fermions. As a consequence, all entries of $\breve{G}$ become two by two matrices.

To derive a kinetic equation, it is useful to introduce Wigner mixed coordinates. To this end we perform a Fourier transform with respect to both space $\left(\mathbf{r}_{1}-\mathbf{r}_{2}\right)$ and time $\left(t_{1}-t_{2}\right)$ relative coordinates

$$
\check{G}(\mathbf{p}, \epsilon ; \mathbf{r}, t)=\int \mathrm{d}\left(t_{1}-t_{2}\right) \int \mathrm{d}\left(\mathbf{r}_{1}-\mathbf{r}_{2}\right) \check{G}\left(\mathbf{r}_{1}, t_{1} ; \mathbf{r}_{2}, t_{2}\right) e^{i\left[\epsilon\left(t_{1}-t_{2}\right)-\mathbf{p} \cdot\left(\mathbf{r}_{1}-\mathbf{r}_{2}\right)\right]} .
$$

The first step in the standard derivation of the kinetic equation is the leftright subtracted Dyson equation

$$
\left[\check{G}_{0}^{-1}\left(x_{1}, x_{3}\right), \check{G}\left(x_{3}, x_{2}\right)\right]=0,
$$

where we have used space-time coordinates $x_{1} \equiv\left(\mathbf{r}_{1}, t_{1}\right)$ etc. In Eq. (3), the symbol $\otimes$ implies integration over $x_{3}$ and matrix multiplication both in Keldysh and spin (if any) spaces. Furthermore

$$
\check{G}_{0}^{-1}\left(x_{1}, x_{3}\right)=\left(i \partial_{t_{1}}-H\right) \delta\left(x_{1}-x_{3}\right),
$$

where $H$ is the Hamiltonian operator. In these lecture notes we do not consider electron-electron interaction. Quite generally the Hamiltonian operator takes the form

$$
H=\frac{\left(-i \nabla_{\mathbf{r}}+e \mathbf{A}(\mathbf{r}, t)\right)^{2}}{2 m}-e \Phi(\mathbf{r}, t)+V(\mathbf{r}) .
$$

Here $e=|e|$ and we have assumed negatively charged particles. In Eq. (5) the scalar and vector potential have a two by two matrix structure, which can be shown by expanding them in the basis of the Pauli matrices

$$
\Phi=\Phi^{0} \sigma^{0}+\Phi^{a} \frac{\sigma^{a}}{2}, \mathbf{A}=\mathbf{A}^{0} \sigma^{0}+\mathbf{A}^{a} \frac{\sigma^{a}}{2}, a=x, y, z,
$$

and summation over the repeated indices is understood. The $\sigma^{0}$ components are the electromagnetic scalar and vector potentials associated with the $\mathrm{U}(1)$ gauge invariance. $V(\mathbf{r})$ describes the disorder potential due to impurities and defects. In this section we set $V(\mathbf{r})=0$ and postpone its discussion to the following section. The $\sigma^{a}$-components are an $\mathrm{SU}(2)$ gauge field, whose scalar and vector components can be used to respectively describe a Zeeman/exchange term and SOC - in our case Rashba SOC, as will be shown in Sec. 4. For the time being, we do not consider a specific form of the $\mathrm{SU}(2)$ gauge field $\left(\Phi^{a}, \mathbf{A}^{a}\right)$.

The goal of a kinetic equation is to describe non-equilibrium phenomena. In general this is a formidable task. However, for close-to-equilibrium phenomena or for non-equilibrium ones occurring on scales large compared to 
microscopic ones, it is possible to derive an effective kinetic equation by means of the so-called gradient expansion. The idea is based on the observation that under specific circumstances the Green function varies fast with respect to the relative coordinate $x_{1}-x_{2}$ and much more slowly with respect to the center-of-mass one $\left(x_{1}+x_{2}\right) / 2$. In equilibrium, for a translationally invariant system, the Green function does not depend on the center-of-mass coordinate at all.

To understand how the gradient expansion works, consider the convolution of two quantities

$$
(A \otimes B)\left(x_{1}, x_{2}\right)=\int \mathrm{d} x_{3} A\left(x_{1}, x_{3}\right) B\left(x_{3}, x_{2}\right),
$$

which can be equivalently expressed as a function of center-of-mass and relative coordinates

$$
\int \mathrm{d} x_{3} A\left(\frac{x_{1}+x_{3}}{2}, x_{1}-x_{3}\right) B\left(\frac{x_{3}+x_{2}}{2}, x_{3}-x_{2}\right) .
$$

Next, replace $x_{1}+x_{3}=x_{1}+x_{2}+x_{3}-x_{2}$ and $x_{3}+x_{2}=x_{1}+x_{2}-\left(x_{1}-x_{3}\right)$ in the first argument of $A$ and $B$, respectively. By Taylor expanding $A$ with respect to $x_{3}-x_{2}$ in its first argument and $B$ with respect to $x_{1}-x_{3}$ in its first argument, after Fourier transforming according to Eq. (2), one gets

$$
A(x, p) B(x, p)+\frac{i}{2}\left(\partial_{\mu} A(x, p)\right)\left(\partial_{p}^{\mu} B(x, p)\right)-\frac{i}{2}\left(\partial_{p}^{\mu} A(x, p)\right)\left(\partial_{\mu} B(x, p)\right), \quad(7)
$$

where we have introduced the compact (relativistic) space-time notations

$$
x^{\mu}=(t, \mathbf{r}), x_{\mu}=(-t, \mathbf{r}), p^{\mu}=(\epsilon, \mathbf{p}), p_{\mu}=(-\epsilon, \mathbf{p})
$$

and

$$
\partial^{\mu} \equiv \frac{\partial}{\partial x_{\mu}}, \partial_{\mu} \equiv \frac{\partial}{\partial x^{\mu}}, \partial_{p}^{\mu} \equiv \frac{\partial}{\partial p_{\mu}}, \partial_{p, \mu} \equiv \frac{\partial}{\partial p^{\mu}}
$$

in a such a way that the product $p^{\mu} x_{\mu}=-\epsilon t+\mathbf{p} \cdot \mathbf{r}$ has the correct Lorentz metrics. Equation (3) acquires then the form

$$
-i\left[\check{G}_{0}^{-1}, \check{G}\right]+\frac{1}{2}\left\{\left(\partial^{\mu} \check{G}_{0}^{-1}\right),\left(\partial_{p, \mu} \check{G}\right)\right\}-\frac{1}{2}\left\{\left(\partial_{p}^{\mu} \check{G}_{0}^{-1}\right),\left(\partial_{\mu} \check{G}\right)\right\}=0 .
$$

The Hamiltonian (5) is invariant under a gauge transformation $O(x)$, which locally rotates the spinor field

$$
\psi^{\prime}(x)=O(x) \psi(x), \psi^{\prime \dagger}(x)=\psi^{\dagger}(x) O^{\dagger}(x), O(x) O^{\dagger}(x)=1 .
$$

The Green function, however, is not locally covariant, i.e. its transformation depends on two distinct space-time points

$$
\check{G}\left(x_{1}, x_{2}\right) \rightarrow O\left(x_{1}\right) \check{G}\left(x_{1}, x_{2}\right) O^{\dagger}\left(x_{2}\right) .
$$


Physical observables, which are locally covariant, are obtained by considering the Green function in the limit of coinciding space-time points. It is then useful to introduce a locally covariant Green function

$$
\check{\tilde{G}}\left(x_{1}, x_{2}\right)=U_{\Gamma}\left(x, x_{1}\right) \check{G}\left(x_{1}, x_{2}\right) U_{\Gamma}\left(x_{2}, x\right)
$$

where

$$
U_{\Gamma}\left(x, x_{1}\right)=\mathcal{P} \exp \left(-i \int_{x_{1}}^{x} e A^{\mu}(y) \mathrm{d} y_{\mu}\right) .
$$

The line integral of the gauge field is referred to as the Wilson line. In Eq. (14) $\mathcal{P}$ is a path-ordering operator and $A^{\mu}=(\Phi, \mathbf{A}), A_{\mu}=(-\Phi, \mathbf{A})$. Since the Wilson line transforms covariantly

$$
U_{\Gamma}\left(x, x_{1}\right) \rightarrow O(x) U_{\Gamma}\left(x, x_{1}\right) O^{\dagger}\left(x_{1}\right),
$$

one easily sees that the covariant Green function $\check{\tilde{G}}$ transforms in a locally covariant way

$$
\check{\tilde{G}}\left(x_{1}, x_{2}\right) \rightarrow O(x) \check{\tilde{G}}\left(x_{1}, x_{2}\right) O^{\dagger}(x) .
$$

When $x_{1}=x_{2}=x$, the locally covariant Green function coincides with the original Green function. By inverting Eq. (13), one can, via Eq. (10), obtain an equation for the locally covariant Green function. Due to the non-Abelian character of the gauge field, Eq. (13) is not easy to handle. In the spirit of the gradient approximation, we assume that $\partial^{\mu} \partial_{\mu, p} \ll 1$. In addition we also assume that $e A^{\mu} \partial_{\mu, p} \ll 1$. This assumption can be justified on physical grounds once an explicit form is assigned to $A$. Under these assumptions, Eq. (13) becomes

$$
\check{\tilde{G}}=\check{G}-\frac{1}{2}\left\{e A^{\mu} \partial_{p, \mu}, \check{G}\right\}
$$

and its inverse

$$
\check{G}=\check{\tilde{G}}+\frac{1}{2}\left\{e A^{\mu} \partial_{p, \mu}, \check{\tilde{G}}\right\}
$$

By using the decomposition

$$
\delta\left(x_{1}-x_{2}\right)=\int \frac{\mathrm{d}^{d+1} p}{(2 \pi)^{d+1}} e^{i p^{\mu}\left(x_{1, \mu}-x_{2, \mu}\right)},
$$

one obtains

$$
\check{G}_{0}^{-1}(x, p)=\epsilon-\frac{(\mathbf{p}+e \mathbf{A}(x))^{2}}{2 m}+e \Phi(x)=\epsilon-\frac{p^{2}}{2 m}-V^{\mu} e A_{\mu}-\frac{e^{2} \mathbf{A}^{2}}{2 m},
$$


from which

$$
\begin{aligned}
\partial_{p}^{\mu} \check{G}_{0}^{-1}(x, p) & =-V^{\mu}-\left(\partial_{p}^{\mu} V^{\nu}\right) e A_{\nu}, \\
\partial^{\mu} \check{G}_{0}^{-1}(x, p) & =-V^{\nu} \partial^{\mu} e A_{\nu} .
\end{aligned}
$$

In the above $V^{\mu}=(1, \mathbf{p} / m)$ is the $d$-current operator, $d$ being the space dimensionality. In the second equation of (21) we neglected the term $\partial^{\mu} e^{2} \mathbf{A}^{2} / 2 m=e \mathbf{A} \cdot \partial^{\mu} e \mathbf{A}$ because it gives a small correction to $\mathbf{p} \cdot \partial^{\mu} e \mathbf{A}$ when $\mathbf{p} \sim \mathbf{p}_{F}$. Performing the shift transformation of Eq. (17) in Eq. (20) gives

$$
\tilde{\tilde{G}}_{0}^{-1}=\epsilon-\frac{p^{2}}{2 m} .
$$

We have now all the necessary ingredients to obtain the equation for $\check{\tilde{G}}$. We begin by considering the first term of Eq. (10). By applying the shift transformation of Eq. (17) and expressing $\check{G}$ in terms of $\tilde{\tilde{G}}$ via Eq. (18), we obtain

$$
-i\left(\left[\check{G}_{0}^{-1}, \check{\tilde{G}}\right]-\frac{1}{2}\left\{e A_{\mu}, \partial_{p}^{\mu}\left[\check{G}_{0}^{-1}, \tilde{\tilde{G}}\right]\right\}+\frac{1}{2}\left[\check{G}_{0}^{-1},\left\{e A_{\mu}, \partial_{p}^{\mu} \check{\tilde{G}}\right\}\right]\right) .
$$

By means of the identity $\{A,[B, C]\}-[B,\{A, C\}]=\{[A, B], C\}$ we get

$$
i e V^{\mu}\left(\left[A_{\mu}, \tilde{\tilde{G}}\right]+\frac{1}{2}\left\{e\left[A_{\mu}, A_{\nu}\right], \partial_{p}^{\nu} \tilde{\tilde{G}}\right\}\right) .
$$

As for the second term of Eq. (10)

$$
\frac{1}{2}\left\{\left(\partial^{\mu} \check{G}_{0}^{-1}\right),\left(\partial_{p, \mu} \check{G}\right)\right\} \rightarrow-\frac{e}{2} V^{\mu}\left\{\left(\partial^{\nu} A_{\mu}\right), \partial_{p, \nu} \check{\widetilde{G}}\right\},
$$

where the last step follows by considering the first order of the gradient expansion. Finally, for the last term of Eq. (10)

$$
-\frac{1}{2}\left\{\left(\partial_{p}^{\mu} \check{G}_{0}^{-1}\right),\left(\partial_{\mu} \check{G}\right)\right\} \rightarrow V^{\mu}\left[\partial_{\mu} \check{\tilde{G}}+\frac{1}{2}\left\{\left(e \partial_{\mu} A^{\nu}\right), \partial_{p, \nu} \check{\tilde{G}}\right\}\right],
$$

where we omitted terms $\sim \partial_{\mu} \partial_{p}^{\nu} \tilde{\tilde{G}}$ within the first order accuracy of the gradient expansion. By collecting the results of Eqs.(24-26), the equation for $\tilde{\tilde{G}}$ reads

$$
V^{\mu}\left[\tilde{\partial}_{\mu} \check{\tilde{G}}+\frac{1}{2}\left\{e F_{\mu \nu}, \partial_{p}^{\nu} \check{\tilde{G}}\right\}\right]=0,
$$

where we have introduced the covariant derivative

$$
\tilde{\partial}_{\mu} \tilde{\tilde{G}}=\partial_{\mu} \check{\tilde{G}}+i\left[e A_{\mu}, \tilde{\tilde{G}}\right]
$$


and the field strength

$$
F_{\mu \nu}=\partial_{\mu} A_{\nu}-\partial_{\nu} A_{\mu}+i e\left[A_{\mu}, A_{\nu}\right] .
$$

It is useful at this stage to separate the space and time parts and rewrite Eq. (27) as

$$
\left(\tilde{\partial}_{t}+\frac{\mathbf{p}}{m} \cdot \tilde{\nabla}_{\mathbf{r}}\right) \tilde{\tilde{G}}-\frac{e}{2}\left\{\frac{\mathbf{p}}{m} \cdot \mathbf{E}, \partial_{\epsilon} \tilde{\tilde{G}}\right\}+\frac{1}{2}\left\{\mathbf{F}, \nabla_{\mathbf{p}} \check{\tilde{G}}\right\}=0,
$$

where the generalized Lorentz force reads

$$
\mathbf{F}=-e\left(\mathbf{E}+\frac{\mathbf{p}}{m} \times \mathbf{B}\right)
$$

with the $\mathrm{U}(1) \times \mathrm{SU}(2)$ fields given by

$$
\begin{aligned}
\mathbf{E} & =-\partial_{t} \mathbf{A}-\nabla_{\mathbf{r}} \Phi+i e[\Phi, \mathbf{A}], \\
B_{i} & =\frac{1}{2} \varepsilon_{i j k} F^{j k} .
\end{aligned}
$$

Equation (30) is the quantum kinetic equation. One can integrate over the energy $\epsilon$, corresponding to the equal-time limit, in order to obtain a semiclassical kinetic equation. We define the distribution function as

$$
f(\mathbf{p}, \mathbf{r}, t) \equiv \frac{1}{2}\left[1+\int \frac{\mathrm{d} \epsilon}{2 \pi i} \tilde{G}^{K}(\mathbf{p}, \epsilon ; \mathbf{r}, t)\right],
$$

which is a matrix in spin space, $f=f^{0} \sigma^{0}+f^{a} \sigma^{a}, a=x, y, z$. By taking the Keldysh component of Eq. (30) we get

$$
\left(\tilde{\partial}_{t}+\frac{\mathbf{p}}{m} \cdot \tilde{\nabla}_{\mathbf{r}}\right) f(\mathbf{p}, \mathbf{r}, t)+\frac{1}{2}\left\{\mathbf{F} \cdot \nabla_{\mathbf{p}}, f(\mathbf{p}, \mathbf{r}, t)\right\}=0 .
$$

We have then obtained a generalization of the Boltzmann equation, where space and time derivatives are replaced by the covariant ones and the generalized Lorentz force appears. We may then introduce the density and current by integrating over the momentum

$$
\rho(\mathbf{r}, t)=\sum_{\mathbf{p}} f(\mathbf{p}, \mathbf{r}, t), \mathbf{J}(\mathbf{r}, t)=\sum_{\mathbf{p}} \frac{\mathbf{p}}{m} f(\mathbf{p}, \mathbf{r}, t) .
$$

Hence the integration over the momentum of Eq. (34) leads to a continuitylike equation

$$
\tilde{\partial}_{t} \rho(\mathbf{r}, t)+\tilde{\nabla}_{\mathbf{r}} \cdot \mathbf{J}(\mathbf{r}, t)=0 .
$$

We will use the above equation in Section 4, when discussing the spin Hall and inverse spin galvanic/Edelstein effects in the Rashba model. 


\section{The standard model of disorder and the diffusive approximation}

In this section we consider the effect of disorder due to impurity scattering. According to the standard model of disorder ${ }^{5}$ the potential $V(\mathbf{r})$ is assumed to be a random variable with distribution

$$
\langle V(\mathbf{r})\rangle=0,\left\langle V(\mathbf{r}) V\left(\mathbf{r}^{\prime}\right)\right\rangle=n_{i} v_{0}^{2} \delta\left(\mathbf{r}-\mathbf{r}^{\prime}\right) .
$$

In the above $n_{i}$ is the impurity density and $v_{0}$ is the scattering amplitude. Higher momenta can be present, and indeed they will be needed when considering skew-scattering processes, but it is not necessary to specify them for the time being. Disorder effects can be taken into account in perturbation theory via the inclusion of a self-energy. Equation (3) becomes

$$
\left[\check{G}_{0}^{-1}\left(x_{1}, x_{3}\right)^{\otimes}, \check{G}\left(x_{3}, x_{2}\right)\right]=\left[\check{\Sigma}\left(x_{1}, x_{3}\right)^{\otimes}, \check{G}\left(x_{3}, x_{2}\right)\right] .
$$

The lowest order self-energy due to disorder is given in Fig. 1 and its expression reads

$$
\check{\Sigma}_{0}(p, x)=n_{i} v_{0}^{2} \sum_{\mathbf{p}^{\prime}} \check{G}(p, x) .
$$

Notice that the integration is only on the space component of the $d$ momentum $p^{\mu}=\left(\epsilon, \mathbf{p}^{\prime}\right)$. This is a result of the fact that the scattering is elastic. In order to use the above self-energy, we must transform it to the locally covariant formalism according to the transformation of Eq. (17) and express $\check{G}$ in terms of $\check{\tilde{G}}$ via Eq. (18). This procedure is the same we have followed in the previous section to transform the kinetic equation from the form of Eq. (10) to the form Eq. (27). Since the procedure will also be used later on, let us show it in detail in this simple case. First we notice that

$$
U_{\Gamma}\left(x, x_{1}\right)\left[\check{\Sigma}\left(x_{1}, x_{3}\right)^{\otimes}, \check{G}\left(x_{3}, x_{2}\right)\right] U_{\Gamma}\left(x_{2}, x\right)=\left[\check{\Sigma}\left(x_{1}, x_{3}\right)^{\otimes}, \check{\tilde{G}}\left(x_{3}, x_{2}\right)\right]
$$

after using the unitarity of the Wilson line by inserting

$$
U_{\Gamma}\left(x_{3}, x\right) U_{\Gamma}\left(x, x_{3}\right)=1
$$

between the self-energy and the Green function. The locally covariant selfenergy reads

$$
\check{\tilde{\Sigma}}_{0}=n_{i} v_{0}^{2} \sum_{\mathbf{p}^{\prime}}\left(\check{\tilde{G}}_{\mathbf{p}^{\prime}}+\frac{1}{2}\left\{A^{\mu}\left(\partial_{p^{\prime}, \mu}-\partial_{p, \mu}\right), \check{\tilde{G}}_{\mathbf{p}^{\prime}}\right\}\right)=n_{i} v_{0}^{2} \sum_{\mathbf{p}^{\prime}} \check{\tilde{G}}_{\mathbf{p}^{\prime}}
$$

In the above the derivative with respect to $\epsilon$ cancels in the two terms. The derivative with respect to $\mathbf{p}$ vanishes because there is no dependence on 
p. Finally, the derivative with respect to $\mathbf{p}^{\prime}$ can be integrated giving at most a constant, which can be discarded. As a result, the locally covariant self-energy has the same functional form of the original self-energy. The

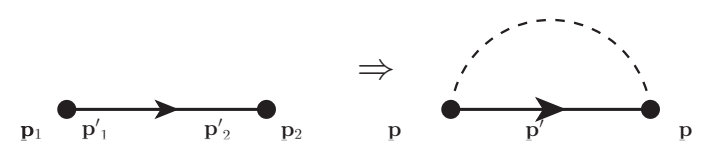

Fig. 1. Self-energy diagram to second order in the impurity potential (black dot vertex). The diagram on the left is before the impurity average, which is carried in the diagram on the right as a dashed line connecting the two impurity insertions. Notice that the impurity average in momentum space yields $\left\langle V\left(\mathbf{p}_{1}-\mathbf{p}_{1}^{\prime}\right) V\left(\mathbf{p}_{2}^{\prime}-\mathbf{p}_{2}\right)\right\rangle=n_{i} v_{0}^{2} \delta\left(\mathbf{p}_{1}-\mathbf{p}_{1}^{\prime}+\right.$ $\left.\mathbf{p}_{2}^{\prime}-\mathbf{p}_{2}\right)$.

Keldysh component of the collision integral then reads

$$
\tilde{I}=-i\left[\check{\tilde{\Sigma}}, \check{\tilde{G}}_{\mathbf{p}}\right]^{K}=-i n_{i} v_{0}^{2} \sum_{\mathbf{p}^{\prime}}\left(\left(\tilde{G}_{\mathbf{p}^{\prime}}^{R}-\tilde{G}_{\mathbf{p}^{\prime}}^{A}\right) \tilde{G}_{\mathbf{p}}^{K}-\left(\tilde{G}_{\mathbf{p}}^{R}-\tilde{G}_{\mathbf{p}}^{A}\right) \tilde{G}_{\mathbf{p}^{\prime}}^{K}\right) .
$$

Note that the $\mathrm{SU}(2)$ shifted retarded and advanced Green functions have no spin structure and therefore commute with the Keldysh Green function. For weak scattering, one can ignore the broadening of the energy levels in the retarded and advanced Green function and use

$$
\tilde{G}_{\mathbf{p}}^{R}-\tilde{G}_{\mathbf{p}}^{A}=-2 \pi i \delta\left(\epsilon-\epsilon_{\mathbf{p}}\right), \tilde{G}_{\mathbf{p}}^{K}=-2 \pi i \delta\left(\epsilon-\epsilon_{\mathbf{p}}\right)(1-2 f(\mathbf{p}, \mathbf{r}, t)) .
$$

As a result, Eq. (34) is no longer collisionless and becomes

$$
\left(\tilde{\partial}_{t}+\frac{\mathbf{p}}{m} \cdot \tilde{\nabla}_{\mathbf{r}}\right) f(\mathbf{p}, \mathbf{r}, t)-\frac{e}{2}\left\{\left(\mathbf{E}+\frac{\mathbf{p}}{m} \times \mathbf{B}\right) \cdot \nabla_{\mathbf{p}}, f(\mathbf{p}, \mathbf{r}, t)\right\}=I[f],
$$

with the collision integral being

$$
I[f]=-2 \pi n_{i} v_{0}^{2} \sum_{\mathbf{p}^{\prime}} \delta\left(\epsilon_{\mathbf{p}}-\epsilon_{\mathbf{p}^{\prime}}\right)\left(f(\mathbf{p}, \mathbf{r}, t)-f\left(\mathbf{p}^{\prime}, \mathbf{r}, t\right)\right) .
$$

It is appropriate in the final part of this section to obtain the solution of the Boltzmann equation Eq. (44) in the diffusive approximation. First we notice that, by integration over the momentum $\mathbf{p}$, the collision integral $I$ vanishes reproducing the continuity equation (36) with density and current defined in Eq. (35). In the diffusive approximation we expand the distribution function in spherical harmonics

$$
f(\mathbf{p}, \mathbf{r}, t)=\langle f\rangle+2 \hat{\mathbf{p}} \cdot \mathbf{f}+\ldots
$$


and keep terms up to the p-wave symmetry. In the above $\langle\ldots\rangle$ indicates the integration over the directions of the momentum. We perform the evaluation in two space dimensions having in mind the application of the theory to the 2DEG. By defining the momentum relaxation time ${ }^{\mathrm{a}}$

$$
\frac{1}{\tau}=2 \pi N_{0} n_{i} v_{0}^{2}
$$

with the density of states $N_{0}=m /(2 \pi)$ the collision integral becomes

$$
I[f]=-\frac{1}{\tau} 2 \hat{\mathbf{p}} \cdot \mathbf{f} .
$$

In the diffusive approximation we consider $\omega \tau \ll 1$ and $v_{F} q \tau \ll 1$, where $\omega$ and $q$ are typical energy and momentum scales. For instance, $\omega$ can be the magnitude of an externally applied magnetic field. We multiply Eq. (44) by $\hat{\mathbf{p}}$ and integrate over the angle $\phi$ with $\hat{\mathbf{p}}=(\cos \phi, \sin \phi)$. We get

$$
-\frac{1}{\tau} \mathbf{f}=\frac{p}{2 m} \tilde{\nabla}_{\mathbf{r}}\langle f\rangle-\frac{e}{2}\left\langle\left\{\hat{\mathbf{p}} \mathbf{E} \cdot \nabla_{\mathbf{p}},\langle f\rangle\right\}\right\rangle-\frac{e}{2 m}\left\langle\left\{\hat{\mathbf{p}}\left(\mathbf{p} \times \mathbf{B} \cdot \nabla_{\mathbf{p}}\right), 2 \hat{\mathbf{p}} \cdot \mathbf{f}\right\}\right\rangle .
$$

The first term, keeping in mind Eq. (35) for the current, represents the diffusive contribution including the additional part due to the $\mathrm{SU}(2)$ gauge field. Due to the covariant nature of the derivative, such a term differs from zero even in uniform circumstances. The second term yields the usual drift contribution, whereas the third one gives rise to a Hall contribution. The gradient with respect to the momentum can be split as $\nabla_{\mathbf{p}}=\hat{\mathbf{p}} \partial_{p}-\hat{\boldsymbol{\phi}} \partial_{\phi} / p$ where $\hat{\boldsymbol{\phi}}=(-\sin \phi, \cos \phi)$. Then we get

$$
\mathbf{f}=-\frac{\tau p}{2 m} \tilde{\nabla}_{\mathbf{r}}\langle f\rangle+\frac{e \tau}{4}\left\{\mathbf{E}, \partial_{p}\langle f\rangle\right\}+\frac{e \tau}{2 m}\{\mathbf{B} \times, \mathbf{f}\} .
$$

By using the definitions of density and current in Eq. (36), we may write the expression for the number and spin components as

$$
n=\operatorname{Tr}[\rho], \mathbf{J}^{0}=\operatorname{Tr}[\mathbf{J}], s^{a}=\frac{1}{2} \operatorname{Tr}\left[\sigma^{a} \rho\right], \mathbf{J}^{a}=\frac{1}{2} \operatorname{Tr}\left[\sigma^{a} \mathbf{J}\right] .
$$

To begin with, let us consider the drift term

$\mathbf{J}_{d r}=\sum_{\mathbf{p}} \frac{p}{m} \frac{e \tau}{4}\left\{\mathbf{E}, \partial_{p}\langle f\rangle\right\}=e N_{0} \int \mathrm{d} \epsilon_{\mathbf{p}} D\left(\epsilon_{\mathbf{p}}\right) \frac{1}{2}\left\{\partial_{\epsilon_{\mathbf{p}}}\langle f\rangle, \mathbf{E}\right\}=-\frac{e}{2}\{\sigma(\mu), \mathbf{E}\}$

where $\epsilon_{\mathbf{p}}=p^{2} / 2 m, D\left(\epsilon_{\mathbf{p}}\right)=\tau \epsilon_{\mathbf{p}} / m, \mu=\rho / N_{0}$ is a spin-dependent chemical potential, and $\sigma(\mu)=N_{0} D(\mu)$. In equilibrium, $\rho_{e q}=N_{0} \epsilon_{F}+N_{0} \Phi$ and its 
eigenvalues determine the chemical potentials for up and down electrons. Then

$$
\mathbf{J}_{d r}^{0}=-e N_{0} D^{0} \mathbf{E}^{0}-\frac{e}{2} N_{0} D^{a} \mathbf{E}^{a}, \mathbf{J}_{d r}^{a}=-\frac{e}{4} N_{0} D^{0} \mathbf{E}^{a}-\frac{e}{2} N_{0} D^{a} \mathbf{E}^{0},
$$

with $D^{0}$ and $D^{a}$ defined by $D(\mu)=D^{0}+D^{a} \sigma^{a}$. By expanding around $\epsilon_{F}$, one has $D^{0} \approx D\left(\epsilon_{F}\right)$ and $D^{a} \approx \tau s^{a} /\left(N_{0} m\right)$, and therefore

$$
\sigma(\mu)=N_{0} D\left(\epsilon_{F}\right) \sigma^{0}+\frac{\tau}{m} s^{a} \sigma^{a} .
$$

The diffusion term is obtained by integrating over the momentum the first term of Eq. (50)

$$
\mathbf{J}_{d i f}=-N_{0} \int \mathrm{d} \epsilon_{\mathbf{p}} D\left(\epsilon_{\mathbf{p}}\right) \tilde{\nabla}_{\mathbf{r}}\langle f\rangle=-\frac{1}{2}\left\{D(\mu), \tilde{\nabla}_{\mathbf{r}} \rho\right\} .
$$

The above form of the diffusion term is determined by requiring that in equilibrium it must cancel the drift term according to the Einstein argument. Then

$$
\mathbf{J}_{d i f}^{0}=-D^{0} \nabla_{\mathbf{r}} n-2 D^{a}\left[\tilde{\nabla}_{\mathbf{r}} s\right]^{a}, \mathbf{J}_{d i f}^{a}=-\frac{1}{2} D^{a} \nabla_{\mathbf{r}} n-D^{0}\left[\tilde{\nabla}_{\mathbf{r}} s\right]^{a} .
$$

Finally the Hall term yields

$$
\mathbf{J}_{\text {Hall }}^{0}=\frac{e \tau}{m} \mathbf{B}^{0} \times \mathbf{J}^{0}+\frac{e \tau}{m} \mathbf{B}^{a} \times \mathbf{J}^{a}, \quad \mathbf{J}_{\text {Hall }}^{a}=\frac{e \tau}{m} \mathbf{B}^{0} \times \mathbf{J}^{a}+\frac{e \tau}{4 m} \mathbf{B}^{a} \times \mathbf{J}^{0} .
$$

To summarize, we may write the particle and spin currents as

$$
\begin{aligned}
\mathbf{J}^{0} & =-e N_{0} D^{0} \mathbf{E}^{0}-\frac{e}{2} N_{0} D^{a} \mathbf{E}^{a}-D^{0} \nabla_{\mathbf{r}} n-2 D^{a}\left[\tilde{\nabla}_{\mathbf{r}} s\right]^{a} \\
& +\frac{e \tau}{m} \mathbf{B}^{0} \times \mathbf{J}^{0}+\frac{e \tau}{m} \mathbf{B}^{a} \times \mathbf{J}^{a}
\end{aligned}
$$

and

$$
\begin{aligned}
\mathbf{J}^{a} & =-\frac{e}{4} N_{0} D^{0} \mathbf{E}^{a}-\frac{e}{2} N_{0} D^{a} \mathbf{E}^{0}-\frac{1}{2} D^{a} \nabla_{\mathbf{r}} n-D^{0}\left[\tilde{\nabla}_{\mathbf{r}} s\right]^{a} \\
& +\frac{e \tau}{m} \mathbf{B}^{0} \times \mathbf{J}^{a}+\frac{e \tau}{4 m} \mathbf{B}^{a} \times \mathbf{J}^{0} .
\end{aligned}
$$

The above two equations, together with the continuity-like Eq. (36), will be used in the next section to analyze the spin Hall and Edelstein effect in the disordered Rashba model. 


\section{The disordered Rashba model}

The Rashba Hamiltonian reads ${ }^{6}$

$$
H=\frac{p^{2}}{2 m}+\alpha p_{y} \sigma^{x}-\alpha p_{x} \sigma^{y} .
$$

The only non zero components of the SU(2) gauge field are

$$
e A_{x}^{y}=-2 m \alpha, e A_{y}^{x}=2 m \alpha .
$$

As shown in the previous sections (cf. Eq. (36)), the spin density obeys a continuity-like equation

$$
\tilde{\partial}_{t} s^{a}+\tilde{\nabla}_{\mathbf{r}} \cdot \mathbf{J}^{a}=0
$$

which is deceptively simple. The notable fact in the present theory is that the covariant derivatives defined in Eq. (28) appear also at the level of the effective phenomenological equations, providing an elegant and compact way to derive the equation of motion for the spin density. The explicit expressions of the space and time covariant derivatives of a generic observable $\mathcal{O}^{a}$ read $^{b}$

$$
\begin{aligned}
\tilde{\partial}_{t} \mathcal{O}^{a} & =\partial_{t} \mathcal{O}^{a}+\epsilon_{a b c} e \Phi^{b} \mathcal{O}^{c} \\
\tilde{\nabla}_{i} \mathcal{O}^{a} & =\nabla_{i} \mathcal{O}^{a}-\epsilon_{a b c} e A_{i}^{b} \mathcal{O}^{c} .
\end{aligned}
$$

Equation (62) becomes

$$
\partial_{t} s^{a}+\epsilon_{a b c} e \Phi^{b} s^{c}+\nabla_{i} J_{i}^{a}-\epsilon_{a b c} e A_{i}^{b} J_{i}^{c}=0,
$$

showing that the equation for the spin is not a simple continuity equation, as expected from the non conservation of spin. The second term in Eq. (65) is the standard precession term. The last term of (65) can be made explicit by providing the expression for the spin current $J_{i}^{a}$, where the lower (upper) index indicates the space (spin) component. The expression of $J_{i}^{a}$ was derived via a microscopic theory in the diffusive regime in Eq. (59). The explicit expression reads

$$
J_{i}^{a}=-\frac{e \tau}{m} s^{a} E_{i}+D \epsilon_{a b c} e A_{i}^{b} s^{c}-\frac{e \tau}{4 m} \epsilon_{i j k} J_{j} B_{k}^{a}-\frac{e D N_{0}}{2} E_{i}^{a},
$$

where $D=D\left(\epsilon_{F}\right)$ is the diffusion constant. Let us apply Eqs. (65-66) to the Rashba model defined by Eq. (61) in the presence of an applied electric field along the x direction $E_{x}$. To linear order in the electric field, the first term of Eq. (66) does not contribute in a paramagnetic system. Also by

\footnotetext{
$\overline{\mathrm{b}} \epsilon_{a b c}$ is the fully antisymmetric Ricci tensor.
} 
using Eq. (61) in the expressions of the fields of Eq. (32) we get that the $\mathrm{SU}(2)$ electric field vanishes $E_{i}^{a}=0$ and that the only non zero component of the $\mathrm{SU}(2)$ magnetic field reads

$$
e B_{z}^{z}=-(2 m \alpha)^{2}
$$

Because the electric field is uniform, we may ignore the space derivative and obtain the explicit form of Eq. (65)

$$
\begin{aligned}
& \partial_{t} s^{x}=-2 m \alpha J_{x}^{z} \\
& \partial_{t} s^{y}=-2 m \alpha J_{y}^{z} \\
& \partial_{t} s^{z}=+2 m \alpha J_{y}^{y}+2 m \alpha J_{x}^{x}
\end{aligned}
$$

with the associated expressions for the spin currents

$$
\begin{aligned}
J_{x}^{z} & =2 m \alpha D s^{x} \\
J_{y}^{z} & =2 m \alpha D s^{y}+\theta_{S H}^{i n t} J_{x}^{0} \\
J_{x}^{x}=J_{y}^{y} & =-2 m \alpha D s^{z},
\end{aligned}
$$

where $J_{x}^{0}=-e \sigma\left(\epsilon_{F}\right) E_{x}$ is the charge current and $\theta_{S H}^{i n t}$ is the spin Hall angle for intrinsic SOC

$$
\theta_{S H}^{i n t}=-m \tau \alpha^{2} .
$$

Insertion of Eqs. (71-73) into (68-70) gives the Bloch equations

$$
\begin{aligned}
& \partial_{t} s^{x}=-\frac{1}{\tau_{D P}} s^{x} \\
& \partial_{t} s^{y}=-\frac{1}{\tau_{D P}}\left(s^{y}-s_{0}\right) \\
& \partial_{t} s^{z}=-\frac{2}{\tau_{D P}} s^{x}
\end{aligned}
$$

where the Dyakonov-Perel relaxation time is given by

$$
\tau_{D P}^{-1}=(2 m \alpha)^{2} D
$$

and the current-induced spin polarization is given by

$$
s_{0}=-e N_{0} \alpha \tau E_{x} .
$$

In the static limit the solution of the Bloch equations yields an in-plane spin polarization perpendicular to the electric field $s^{y}=s^{0}$, with $s^{x}=s^{z}=0$. This is known as the Edelstein ${ }^{7,8}$ or inverse spin-galvanic effect ${ }^{9,10}$. The vanishing of the time derivative implies, via Eq. (69), the vanishing of the spin current $J_{y}^{z}$ associated to the spin Hall effect. This vanishing occurs 
thanks to the exact compensation of the two contributions appearing in Eq. (72). c

The above analysis can be extended in the presence of a thermal gradient. More precisely, we derive $s^{y}$ in terms of a stationary thermal gradient along the $x$-direction, $\nabla_{x} T$, in the absence of any additional external fields. ${ }^{12}$ However, in an experiment one would still measure an electric field $E_{x}$ due to a gradient in the chemical potential, $\nabla_{x} \mu$, resulting from an imbalance of the charge carriers due to the thermal gradient. For this, we shall first consider the trace of Eq. (50):

$$
\mathbf{f}^{0}=-\frac{\tau p}{2 m} \nabla\left\langle f^{0}\right\rangle,
$$

where we can approximate $\nabla\left\langle f^{0}\right\rangle$ as the Fermi function $f^{e q}$, giving us

$$
f_{x}^{0}=-\frac{\tau p}{2 m}\left(\frac{\epsilon-\mu}{T} \nabla_{x} T+\nabla_{x} \mu\right)\left(-\frac{\partial f^{e q}}{\partial \epsilon}\right)
$$

for the $x$-component of Eq. (80). With use of the Sommerfeld expansion

$$
\int d \epsilon g(\epsilon)\left(-\frac{\partial f^{e q}}{\partial \epsilon}\right)=g(\mu)+\left.\frac{\pi^{2}}{6}\left(k_{B} T\right)^{2} \frac{\partial^{2} g}{\partial \epsilon^{2}}\right|_{\epsilon=\mu},
$$

where $g(\epsilon)$ is an arbitrary energy dependent function, we end up with the particle current in the $x$-direction as follows:

$$
J_{x}^{0}=-\frac{2 \tau N_{0}}{m}\left[\frac{\pi^{2}}{3} \frac{\left(k_{B} T\right)^{2}}{T} \nabla_{x} T+\mu \nabla_{x} \mu\right] .
$$

We shall consider an open circuit along $x$-direction, i.e., a vanishing particle current $J_{x}^{0}=0$. Then, we can express the electric field one would measure in an experiment as

$$
E_{x}=\frac{1}{e} \nabla_{x} \mu=S \nabla_{x} T
$$

where $S=-\left(\pi k_{B}\right)^{2} T /(3 e \mu)$ is the Seebeck coefficient. After having analyzed the charge sector, we consider next the spin sector in order to get an expression for $s^{y}$. We start by multiplying Eq. (50) with $\sigma^{z}$ and perform the trace. The $y$-component reads

$$
f_{y}^{z}=2 p \tau \alpha\left\langle f^{y}\right\rangle+\frac{e \tau}{m} B_{z}^{z} f_{x}^{0} .
$$

\footnotetext{
c To make contact with the diagrammatic Kubo formula approach, we notice that the second term of Eq. (72) corresponds to a bubble-like diagram, whereas the first term describes the so-called vertex corrections. ${ }^{11}$
} 
Note that the form of Eq. (69) doesn't change and since we assume a stationary case we have

$$
J_{y}^{z}=0 \Leftrightarrow f_{y}^{z}=0 .
$$

This implies that there is no spin Nernst effect as no spin Hall effect in the disordered Rashba model. From Eq. (85), together with Eq. (81) we therefore find

$$
\left\langle f^{y}\right\rangle=\frac{2 \alpha m}{p} f_{x}^{0}=-\alpha \tau\left(\frac{\epsilon-\mu}{T} \nabla_{x} T+\nabla_{x} \mu\right)\left(-\frac{\partial f^{e q}}{\partial \epsilon}\right) .
$$

From the form of the latter equation and with use of the Sommerfeld expansion, Eq. (82), it is clear that we can express the $y$ spin polarization as

$$
s^{y}=P_{s T} \nabla_{x} T+P_{s E} E_{x},
$$

where $P_{s T}$ can be written in a Mott-like form:

$$
P_{s T}=-S \mu \frac{\partial P_{s E}}{\partial \mu} .
$$

Here, we find $P_{s E}=-\alpha \tau e N_{0}$, consistent with Eq. (76). This results in a vanishing $P_{s T}$ since $P_{s E}$ is independent of $\mu$. We express $E_{x}$ in terms of $\nabla_{x} T$ with use of Eq. (84) and end up with

$$
s^{y}=-\alpha \tau e N_{0} S \nabla_{x} T,
$$

describing the thermal Edelstein effect in the disordered Rashba model.

\section{The impurity-induced spin-orbit coupling: swapping and side jump mechanisms}

In this and following sections we consider the extrinsic SOC due to impurity scattering described by the Hamiltonian

$$
H_{e x t, s o}=-\frac{\lambda_{0}^{2}}{4} \boldsymbol{\sigma} \times \nabla V(\mathbf{r}) \cdot \mathbf{p},
$$

where $\lambda_{0}$ is the effective Compton wave length ${ }^{13,14}$. In developing the perturbation theory in the impurity potential we must now use the lowest order scattering amplitude

$$
S_{\mathbf{p}^{\prime}, \mathbf{p}^{\prime \prime}}=V_{\mathbf{p}^{\prime}-\mathbf{p}^{\prime \prime}}\left[1-\frac{i \lambda_{0}^{2}}{4} \mathbf{p}^{\prime} \times \mathbf{p}^{\prime \prime} \cdot \boldsymbol{\sigma}\right]
$$

with

$$
\left\langle V_{\mathbf{q}_{1}} V_{\mathbf{q}_{2}}\right\rangle=n_{i} v_{0}^{2} \delta\left(\mathbf{q}_{1}+\mathbf{q}_{2}\right) .
$$



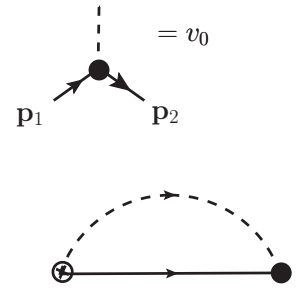
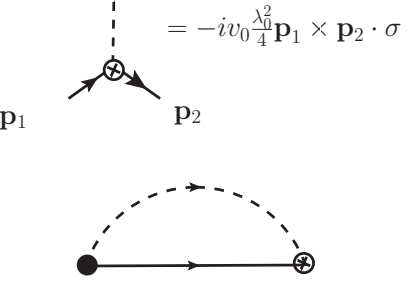

Fig. 2. In the top line the impurity insertion without (black dot vertex) and with (crossed dot vertex) spin-orbit coupling. In the bottom line the two diagrams to first order in the spin-orbit coupling $\lambda_{0}^{2}$.

To zeroth order in $\lambda_{0}^{2}$, we have the diagram of Fig. 1, which has been studied in the previous section. To first order in $\lambda_{0}^{2}$, we must consider the two diagrams of Fig. 2. Here an empty crossed dot stands for the part of the scattering amplitude with the spin-orbit coupling. Let us evaluate these diagrams step by step. Before the impurity average (indicated as $\langle\ldots\rangle$ ), the expression for the two diagrams reads

$$
\begin{aligned}
\check{\Sigma}_{1, \mathbf{p}^{\prime}, \mathbf{p}^{\prime \prime}} & =-i \frac{\lambda_{0}^{2}}{4} \sum_{\mathbf{p}_{1}, \mathbf{p}_{2}}\left\langleV _ { \mathbf { p } ^ { \prime } - \mathbf { p } _ { 1 } } \left(\mathbf{p}^{\prime} \times \mathbf{p}_{1} \cdot \boldsymbol{\sigma} \check{G}_{\mathbf{p}_{1}, \mathbf{p}_{2}}\right.\right. \\
& \left.\left.+\check{G}_{\mathbf{p}_{1}, \mathbf{p}_{2}} \mathbf{p}_{2} \times \mathbf{p}^{\prime \prime} \cdot \boldsymbol{\sigma}\right) V_{\mathbf{p}_{2}-\mathbf{p}^{\prime \prime}}\right\rangle .
\end{aligned}
$$

In the above, $\check{G}_{\mathbf{p}_{1}, \mathbf{p}_{2}}$ is the Fourier transform with respect to the space arguments $\mathbf{r}_{1}$ and $\mathbf{r}_{2}$ of $\check{G}\left(\mathbf{r}_{1}, \mathbf{r}_{2}\right)$. We do not mention explicitly here the time arguments for the sake of simplicity. Performing the impurity average one obtains $\mathbf{p}^{\prime}-\mathbf{p}_{1}=\mathbf{p}^{\prime \prime}-\mathbf{p}_{2}$. It is convenient then to define momenta as

$$
\mathbf{p}=\frac{\mathbf{p}^{\prime}+\mathbf{p}^{\prime \prime}}{2}, \mathbf{q}=\mathbf{p}^{\prime}-\mathbf{p}^{\prime \prime}=\mathbf{p}_{1}-\mathbf{p}_{2}, \tilde{\mathbf{p}}=\frac{\mathbf{p}_{1}+\mathbf{p}_{2}}{2}
$$

in such a way that $\mathbf{p}$ and $\tilde{\mathbf{p}}$ correspond to the momentum of the mixed Wigner representation introduced previously. The momentum $\mathbf{q}$ instead is the variable conjugated to the center-of-mass coordinate $\mathbf{r}$ by Fourier transform. We then get the impurity-averaged expression of the two firstorder diagrams

$$
\begin{aligned}
\check{\Sigma}_{1}(\mathbf{p}, \mathbf{q}) & =-i \frac{\lambda_{0}^{2}}{4} n_{i} v_{0}^{2} \sum_{\tilde{\mathbf{p}}}\left[\left(\mathbf{p}+\frac{\mathbf{q}}{2}\right) \times\left(\tilde{\mathbf{p}}+\frac{\mathbf{q}}{2}\right) \cdot \boldsymbol{\sigma} \check{G}(\tilde{\mathbf{p}}, \mathbf{q})\right. \\
& \left.+\check{G}(\tilde{\mathbf{p}}, \mathbf{q})\left(\tilde{\mathbf{p}}-\frac{\mathbf{q}}{2}\right) \times\left(\mathbf{p}-\frac{\mathbf{q}}{2}\right) \cdot \boldsymbol{\sigma}\right] .
\end{aligned}
$$


The above expression can be divided into three terms

$$
\begin{aligned}
& \left.\check{\Sigma}_{1, a}(\mathbf{p}, \mathbf{q})\right)=-i \frac{\lambda_{0}^{2}}{4} n_{i} v_{0}^{2} \sum_{\tilde{\mathbf{p}}}[\mathbf{p} \times \tilde{\mathbf{p}} \cdot \boldsymbol{\sigma}, \check{G}(\tilde{\mathbf{p}}, \mathbf{q})] \\
& \left.\check{\Sigma}_{1, b}(\mathbf{p}, \mathbf{q})\right)=-i \frac{\lambda_{0}^{2}}{8} n_{i} v_{0}^{2} \sum_{\tilde{\mathbf{p}}}\{\mathbf{p} \times \mathbf{q} \cdot \boldsymbol{\sigma}, \check{G}(\tilde{\mathbf{p}}, \mathbf{q})\} \\
& \left.\check{\Sigma}_{1, c}(\mathbf{p}, \mathbf{q})\right)=i \frac{\lambda_{0}^{2}}{8} n_{i} v_{0}^{2} \sum_{\tilde{\mathbf{p}}}\{\tilde{\mathbf{p}} \times \mathbf{q} \cdot \boldsymbol{\sigma}, \check{G}(\tilde{\mathbf{p}}, \mathbf{q})\} .
\end{aligned}
$$

One can Fourier transform back to the center-of-mass coordinate $\mathbf{r}$ and re-label $\tilde{\mathbf{p}} \rightarrow \mathbf{p}^{\prime}$

$$
\begin{aligned}
& \left.\check{\Sigma}_{1, a}(\mathbf{p}, \mathbf{r})\right)=-i \frac{\lambda_{0}^{2}}{4} n_{i} v_{0}^{2} \sum_{\mathbf{p}^{\prime}}\left[\mathbf{p} \times \mathbf{p}^{\prime} \cdot \boldsymbol{\sigma}, \check{G}\left(\mathbf{p}^{\prime}, \mathbf{r}\right)\right] \\
& \left.\check{\Sigma}_{1, b}(\mathbf{p}, \mathbf{r})\right)=-\nabla_{\mathbf{r}} \cdot \frac{\lambda_{0}^{2}}{8} n_{i} v_{0}^{2} \sum_{\mathbf{p}^{\prime}}\left\{\boldsymbol{\sigma} \times \mathbf{p}, \check{G}\left(\mathbf{p}^{\prime}, \mathbf{r}\right)\right\} \\
& \left.\check{\Sigma}_{1, c}(\mathbf{p}, \mathbf{r})\right)=\nabla_{\mathbf{r}} \cdot \frac{\lambda_{0}^{2}}{8} n_{i} v_{0}^{2} \sum_{\mathbf{p}^{\prime}}\left\{\boldsymbol{\sigma} \times \mathbf{p}^{\prime}, \check{G}\left(\mathbf{p}^{\prime}, \mathbf{r}\right)\right\} .
\end{aligned}
$$

Equations (100-102) are the final expression for the diagrams of the second line of Fig. 2. The first term $\check{\Sigma}_{1, a}$, as we will see, describes the swapping of spin currents under scattering ${ }^{15}$. The other two terms, $\check{\Sigma}_{1, b}$ and $\check{\Sigma}_{1, c}$, are written as a divergence. As a consequence, when they are inserted in the collision integral of the kinetic equation, they lead to a correction of the velocity operator and hence describe the so-called side-jump mechanism ${ }^{16}$. Until now we have not yet considered the effect of the gauge fields on the extrinsic SOC. To do it, we must transform the above derived self-energy to the locally covariant form. Let us first examine the $\mathrm{U}(1)$ gauge field corresponding to a static electric field $\mathbf{E}^{0}=-\nabla_{\mathbf{r}} \Phi^{0}(\mathbf{r})$. The shift of the gradient of the Green function yields

$$
\begin{aligned}
\nabla_{\mathbf{r}} \check{\tilde{G}(\mathbf{p}, \mathbf{r})} & =\nabla_{\mathbf{r}} \check{G}(\mathbf{p}, \mathbf{r})-\frac{1}{2}\left\{e \Phi^{0} \partial_{\epsilon}, \nabla_{\mathbf{r}} \check{G}(\mathbf{p}, \mathbf{r})\right\} \\
& =\nabla_{\mathbf{r}} \check{\tilde{G}}(\mathbf{p}, \mathbf{r})+\frac{1}{2} \nabla_{\mathbf{r}}\left\{e \Phi^{0} \partial_{\epsilon}, \check{\tilde{G}}(\mathbf{p}, \mathbf{r})\right\}-\frac{1}{2}\left\{e \Phi^{0} \partial_{\epsilon}, \nabla_{\mathbf{r}} \check{\tilde{G}}(\mathbf{p}, \mathbf{r})\right\} \\
& =\nabla_{\mathbf{r}} \check{\tilde{G}}(\mathbf{p}, \mathbf{r})-e \mathbf{E}^{0} \partial_{\epsilon} \tilde{\tilde{G}}(\mathbf{p}, \mathbf{r}) .
\end{aligned}
$$


As a result we get from Eqs.(101-102) two more terms

$$
\begin{aligned}
& \left.\delta \check{\Sigma}_{1, b}(\mathbf{p}, \mathbf{r})\right)=e \mathbf{E}^{0} \cdot \frac{\lambda_{0}^{2}}{8} n_{i} v_{0}^{2} \sum_{\mathbf{p}^{\prime}}\left\{\boldsymbol{\sigma} \times \mathbf{p}, \partial_{\epsilon} \check{\tilde{G}}\left(\mathbf{p}^{\prime}, \mathbf{r}\right)\right\} \\
& \left.\delta \check{\Sigma}_{1, c}(\mathbf{p}, \mathbf{r})\right)=-e \mathbf{E}^{0} \cdot \frac{\lambda_{0}^{2}}{8} n_{i} v_{0}^{2} \sum_{\mathbf{p}^{\prime}}\left\{\boldsymbol{\sigma} \times \mathbf{p}^{\prime}, \partial_{\epsilon} \check{\tilde{G}}\left(\mathbf{p}^{\prime}, \mathbf{r}\right)\right\} .
\end{aligned}
$$

Let us transform Eq. (100) to the locally covariant form

$$
\begin{aligned}
\check{\tilde{\Sigma}}_{1, a}(\mathbf{p}, \mathbf{r}) & =-i \frac{\lambda_{0}^{2}}{4} n_{i} v_{0}^{2} \sum_{\mathbf{p}^{\prime}}\left[\mathbf{p} \times \mathbf{p}^{\prime} \cdot \boldsymbol{\sigma}, \check{\tilde{G}}\left(\mathbf{p}^{\prime}, \mathbf{r}\right)\right] \\
& +i \frac{\lambda_{0}^{2}}{4} n_{i} v_{0}^{2} \sum_{\mathbf{p}^{\prime}} \frac{1}{2}\left[\mathbf{p} \times \mathbf{p}^{\prime} \cdot \boldsymbol{\sigma},\left\{e \mathbf{A} \cdot \nabla_{\mathbf{p}^{\prime}}, \check{\tilde{G}}\left(\mathbf{p}^{\prime}, \mathbf{r}\right)\right\}\right] \\
& -i \frac{\lambda_{0}^{2}}{4} n_{i} v_{0}^{2} \sum_{\mathbf{p}^{\prime}} \frac{1}{2}\left\{e \mathbf{A} \cdot \nabla_{\mathbf{p}},\left[\mathbf{p} \times \mathbf{p}^{\prime} \cdot \boldsymbol{\sigma}, \check{\tilde{G}}\left(\mathbf{p}^{\prime}, \mathbf{r}\right)\right]\right\},
\end{aligned}
$$

which can be rewritten as

$$
\begin{aligned}
\check{\tilde{\Sigma}}_{1, a}(\mathbf{p}, \mathbf{r}) & =-i \frac{\lambda_{0}^{2}}{4} n_{i} v_{0}^{2} \sum_{\mathbf{p}^{\prime}}\left[\mathbf{p} \times \mathbf{p}^{\prime} \cdot \boldsymbol{\sigma}, \tilde{\tilde{G}}\left(\mathbf{p}^{\prime}, \mathbf{r}\right)\right] \\
& +i \frac{\lambda_{0}^{2}}{4} n_{i} v_{0}^{2} \sum_{\mathbf{p}^{\prime}} \frac{1}{2}\left[e \mathbf{A} ;\left\{\boldsymbol{\sigma} \times \mathbf{p}, \check{\tilde{G}}\left(\mathbf{p}^{\prime}, \mathbf{r}\right)\right\}\right] \\
& -i \frac{\lambda_{0}^{2}}{4} n_{i} v_{0}^{2} \sum_{\mathbf{p}^{\prime}} \frac{1}{2}\left\{\boldsymbol{\sigma} \times \mathbf{p}^{\prime} ;\left[e \mathbf{A}, \check{\tilde{G}}\left(\mathbf{p}^{\prime}, \mathbf{r}\right)\right]\right\} .
\end{aligned}
$$

As a result, the contribution of the diagrams of Fig. 2 can be written as

$$
\begin{aligned}
& \check{\tilde{\Sigma}}_{1, a}^{S C S}=-i \frac{\lambda_{0}^{2}}{4} n_{i} v_{0}^{2} \sum_{\mathbf{p}^{\prime}}\left[\mathbf{p} \times \mathbf{p}^{\prime} \cdot \boldsymbol{\sigma}, \check{\tilde{G}}\left(\mathbf{p}^{\prime}, \mathbf{r}\right)\right] \\
& \check{\tilde{\Sigma}}_{1, b}^{S J}=-\frac{\lambda_{0}^{2}}{8} n_{i} v_{0}^{2} \sum_{\mathbf{p}^{\prime}} \tilde{\nabla}_{\mathbf{r}}\left\{\boldsymbol{\sigma} \times \mathbf{p}, \check{\tilde{G}}\left(\mathbf{p}^{\prime}, \mathbf{r}\right)\right\} \\
& \check{\tilde{\Sigma}}_{1, c}^{S J}=\frac{\lambda_{0}^{2}}{8} n_{i} v_{0}^{2} \sum_{\mathbf{p}^{\prime}}\left\{\boldsymbol{\sigma} \times \mathbf{p}^{\prime}, \tilde{\nabla} \mathbf{r} \tilde{\tilde{G}}\left(\mathbf{p}^{\prime}, \mathbf{r}\right)\right\} .
\end{aligned}
$$

where $\tilde{\nabla}_{\mathbf{r}}=\nabla_{\mathbf{r}}-e \mathbf{E}^{0} \partial_{\epsilon}+i[e \mathbf{A}, \ldots]$. A few comments are needed at this point. As already mentioned, the term $\underset{\tilde{\Sigma}}{\tilde{\Sigma}} S C S$ describes the spin current swapping (SCS) defined by

$$
J_{i}^{a}=\kappa\left[J_{a}^{i}-\delta_{i a} \sum_{l} J_{l}^{l}\right] .
$$


This is evident in the fact that this term contains the vector product $\mathbf{p} \times \mathbf{p}^{\prime}$ of the momenta before and after the scattering from the impurity. The presence of both momenta yields the coupling of the currents of incoming and outgoing particles. The other two terms $\tilde{\tilde{\Sigma}}_{1, b}^{S J}$ and $\tilde{\tilde{\Sigma}}_{1, c}^{S J}$ describe the so-called side jump (SJ) effect. This is evident in both terms, which show the operator $\boldsymbol{\sigma} \times\left(\mathbf{p}-\mathbf{p}^{\prime}\right)$. By a semiclassical analysis one can show that $\Delta \mathbf{r} \equiv-\left(\lambda_{0}^{2} / 4\right)\left(\mathbf{p}^{\prime}-\mathbf{p}\right) \times \boldsymbol{\sigma}$ is the side jump shift caused by the SOC to the scattering trajectory of a wave packet. The SJ terms are composed of three parts. The first part is the one under the space derivative sign and can be written as $-\nabla_{\mathbf{r}} \cdot \delta \mathbf{J}^{S J}$, i.e. it describes a modification of the current operator. Eventually this term yields the first one-half of the side jump. The second part, proportional to the electric field, describes how the energy of a scattering particle is affected by the effective dipole energy $\sim e \mathbf{E}^{0} \cdot \Delta \mathbf{r}$ due to the side jump shift of the trajectory. This is the other one-half contribution to the side jump. The third part reconstructs the full covariant derivative in the presence of a SU(2) gauge field $\mathbf{A}=\mathbf{A}^{a} \sigma^{a} / 2$. To first order accuracy in the gradient expansion, we have replaced the $\check{G}$ with $\check{\tilde{G}}$ in the terms where the gradient or the gauge field appear. To make the above comments explicit, we start by noticing that only the Keldysh component appears in Eq. (107) because both $\tilde{G}^{R}$ and $\tilde{G}^{A}$ are proportional to $\sigma^{0}$ and commute with all the Pauli matrices. By considering that the Keldysh component of the collision integral requires $\tilde{\Sigma}^{R} \tilde{G}^{K}-\tilde{G}^{K} \tilde{\Sigma}^{A}-\left(\tilde{G}^{R}-\tilde{G}^{A}\right) \tilde{\Sigma}^{K}$, one obtains ${ }^{17}$ from Eq. (107)

$$
I^{S C S}[f]=-i \frac{\lambda_{0}^{2}}{4} n_{i} v_{0}^{2} \sum_{\mathbf{p}^{\prime}} \delta\left(\epsilon_{\mathbf{p}}-\epsilon_{\mathbf{p}^{\prime}}\right)\left[\boldsymbol{\sigma} \cdot \mathbf{p} \times \mathbf{p}^{\prime}, f_{\mathbf{p}^{\prime}}\right] .
$$

Similarly, for the side jump we define

$$
I^{S J}=-i \int \frac{\mathrm{d} \epsilon}{4 \pi i}\left(-\left(\tilde{G}^{R}-\tilde{G}^{A}\right) \tilde{\Sigma}^{K}+\tilde{\Sigma}^{R} \tilde{G}^{K}-\tilde{G}^{K} \tilde{\Sigma}^{A}\right) \equiv I^{(a)}+I^{(b)} .
$$

Because of the integration over the angle, the retarded and advanced components of Eq. (109) vanish. The retarded component of Eq. (108) reads

$$
\tilde{\Sigma}_{1, b}^{S J, R}=-i 2 \pi \frac{\lambda_{0}^{2}}{8} n_{i} v_{0}^{2} \sum_{\mathbf{p}^{\prime}} \delta\left(\epsilon_{\mathbf{p}}-\epsilon_{\mathbf{p}^{\prime}}\right) \tilde{\nabla}_{\mathbf{r}} \boldsymbol{\sigma} \times \mathbf{p}
$$

and $\tilde{\Sigma}_{1, b}^{S J, A}=-\tilde{\Sigma}_{1, b}^{S J, R}$. As a result, with $h_{\mathbf{p}} \equiv 1-2 f_{\mathbf{p}}$ for brevity,

$$
I^{(b)}=-\frac{\lambda_{0}^{2}}{16} 2 \pi n_{i} v_{0}^{2} \sum_{\mathbf{p}^{\prime}} \delta\left(\epsilon_{\mathbf{p}}-\epsilon_{\mathbf{p}^{\prime}}\right)\left\{\tilde{\nabla}_{\mathbf{r}}(\boldsymbol{\sigma} \times \mathbf{p}), h_{\mathbf{p}}\right\} .
$$


By using again the identity $\{A,[B, C]\}-[B,\{A, C\}]=\{[A, B], C\}$, the Keldysh component of Eq. (109) reads

$$
\tilde{\Sigma}_{1, c}^{S J, K}=\frac{\lambda_{0}^{2}}{8} n_{i} v_{0}^{2} \sum_{\mathbf{p}^{\prime}}\left(\tilde{\nabla}_{\mathbf{r}}\left\{\boldsymbol{\sigma} \times \mathbf{p}^{\prime}, \tilde{G}_{\mathbf{p}^{\prime}}^{K}\right\}-\left\{\tilde{\nabla}_{\mathbf{r}}\left(\boldsymbol{\sigma} \times \mathbf{p}^{\prime}\right), \tilde{G}_{\mathbf{p}^{\prime}}^{K}\right\}\right) .
$$

By combining the last result with Eq. (108) and Eq. (114), one obtains finally

$$
\begin{aligned}
I^{S J}[f] & =-\tilde{\nabla}_{\mathbf{r}} \cdot \frac{\lambda_{0}^{2}}{8} n_{i} v_{0}^{2} \sum_{\mathbf{p}^{\prime}} \delta\left(\epsilon_{\mathbf{p}}-\epsilon_{\mathbf{p}^{\prime}}\right)\left\{\boldsymbol{\sigma} \times\left(\mathbf{p}^{\prime}-\mathbf{p}\right), f_{\mathbf{p}^{\prime}}\right\} \\
& +\frac{\lambda_{0}^{2}}{8} n_{i} v_{0}^{2} \sum_{\mathbf{p}^{\prime}} \delta\left(\epsilon_{\mathbf{p}}-\epsilon_{\mathbf{p}^{\prime}}\right)\left(\left\{\tilde{\nabla}_{\mathbf{r}}\left(\boldsymbol{\sigma} \times \mathbf{p}^{\prime}\right), f_{\mathbf{p}^{\prime}}\right\}-\left\{\tilde{\nabla}_{\mathbf{r}}(\boldsymbol{\sigma} \times \mathbf{p}), f_{\mathbf{p}}\right\}\right) .
\end{aligned}
$$

The first term on the right hand side, under the covariant space derivatiive, defines the modification of the current operator due to the SOC. We emphasize that such anomalous part of the current is subject to the full covariant derivative. Hence the last term in Eq. (65) remains the same. Notice also that the second term in $I^{S J}[f]$, although it does not contribute to the continuity equation, is necessary to make sure that the equilibrium distribution function solves the kinetic equation.

\section{The impurity-induced spin-orbit coupling: skew scattering}

In this section we discuss skew scattering by considering the diagrams of Fig. 3. To understand the meaning of these diagrams, recall that in general, in the presence of $\mathrm{SOC}$, the scattering amplitude reads

$$
S=A+\hat{\mathbf{p}} \times \hat{\mathbf{p}}^{\prime} \cdot \boldsymbol{\sigma} B,
$$

where $\hat{\mathbf{p}}$ and $\hat{\mathbf{p}}^{\prime}$ are unit vectors in the direction of the momentum before and after the scattering event. To lowest order in perturbation theory or Born approximation one has $A=v_{0}$ and $B=-\mathrm{i}\left(\lambda_{0}^{2} p_{F}^{2} / 4\right) v_{0}$ and one recovers Eq. (92). By considering the scattering probability proportional to $|S|^{2}$, one obtains three contributions given by $|A|^{2},|B|^{2}$ and $2 \mathcal{R} e\left(A B^{*}\right) \hat{\mathbf{p}} \times \hat{\mathbf{p}}^{\prime} \cdot \boldsymbol{\sigma}$. Whereas the first two contributions are spin independent and give the total scattering time, the third one represents the so-called skew scattering term according to which electrons with opposite spin are scattered in opposite directions. Clearly, since $A$ and $B$ are out of phase, there is no skew scattering effect to the order of the Born approximation. For it to appear to first order in the spin-orbit coupling constant $\lambda_{0}^{2}, A$ has to be evaluated 


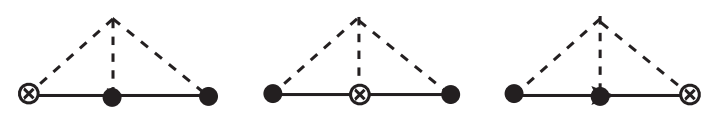

Fig. 3. Third order in $v_{0}$ and first order in $\lambda_{0}^{2}$ diagrams. The skew scattering contribution arises from the first and last diagram.

beyond the Born approximation. The scattering problem can be cast in terms of the Lippman-Schwinger equation

$$
\psi(\mathbf{x})=e^{\mathrm{ik} \cdot \mathbf{x}}+\int \mathrm{d} \mathbf{x}^{\prime} G\left(\mathbf{x}-\mathbf{x}^{\prime}\right) V\left(\mathbf{x}^{\prime}\right) \psi\left(\mathbf{x}^{\prime}\right),
$$

where $G(\mathbf{x})$ is the retarded Green function at fixed energy. From (118) we get

$$
\psi^{(1)}=v_{0} G(\mathbf{x}), A^{(1)}=v_{0} ; \quad \psi^{(2)}=v_{0}^{2} G(\mathbf{0}) G(\mathbf{x}), A^{(2)}=v_{0}^{2} G(\mathbf{0}) .
$$

Notice that only the imaginary part of $A^{(2)}$ is needed. By recalling that Im $G(\mathbf{0})=-\pi N_{0}$, the spin-orbit independent scattering amplitude $A$ up to second order in $v_{0}$ reads

$$
A=v_{0}\left(1-\mathrm{i} \pi N_{0} v_{0}\right) .
$$

The skew scattering contribution will then follow by inserting the modified scattering amplitude (120) into the collision integral of the Boltzmann equation. The same result can, of course, be obtained in quantum field theory using the Green function technique. The latter becomes necessary when one wants to consider skew scattering in the presence of Rashba spinorbit interaction. To this end one has to consider the electron self-energy at least to third order in the scattering potential $v_{0}$. The diagrams of Fig. 3 yield

$$
\begin{aligned}
& \check{\Sigma}_{a}^{S S}=-\frac{i \lambda_{0}^{2}}{4} \sum_{\mathbf{p}_{1}, \mathbf{p}_{2}, \mathbf{p}_{3}, \mathbf{p}_{4}}\left\langle V_{\mathbf{p}^{\prime}-\mathbf{p}_{1}} \check{G}_{\mathbf{p}_{1}, \mathbf{p}_{2}} V_{\mathbf{p}_{2}-\mathbf{p}_{3}} \check{G}_{\mathbf{p}_{3}, \mathbf{p}_{4}} V_{\mathbf{p}_{4}-\mathbf{p}^{\prime \prime}} \mathbf{p}_{4} \times \mathbf{p}^{\prime \prime} \cdot \boldsymbol{\sigma}\right\rangle \\
& \check{\Sigma}_{b}^{S S}=-\frac{i \lambda_{0}^{2}}{4} \sum_{\mathbf{p}_{1}, \mathbf{p}_{2}, \mathbf{p}_{3}, \mathbf{p}_{4}}\left\langle V_{\mathbf{p}^{\prime}-\mathbf{p}_{1}} \check{G}_{\mathbf{p}_{1}, \mathbf{p}_{2}} V_{\mathbf{p}_{2}-\mathbf{p}_{3}} \mathbf{p}_{2} \times \mathbf{p}_{3} \cdot \boldsymbol{\sigma} \check{G}_{\mathbf{p}_{3}, \mathbf{p}_{4}} V_{\mathbf{p}_{4}-\mathbf{p}^{\prime \prime}}\right\rangle \\
& \check{\Sigma}_{c}^{S S}=-\frac{i \lambda_{0}^{2}}{4} \sum_{\mathbf{p}_{1}, \mathbf{p}_{2}, \mathbf{p}_{3}, \mathbf{p}_{4}}\left\langle V_{\mathbf{p}^{\prime}-\mathbf{p}_{1}} \mathbf{p}^{\prime} \times \mathbf{p}_{1} \cdot \boldsymbol{\sigma} \check{G}_{\mathbf{p}_{1}, \mathbf{p}_{2}} V_{\mathbf{p}_{2}-\mathbf{p}_{3}} \check{G}_{\mathbf{p}_{3}, \mathbf{p}_{4}} V_{\mathbf{p}_{4}-\mathbf{p}^{\prime \prime}}\right\rangle
\end{aligned}
$$

By requiring the existence of third moments of the random potential $\left\langle V\left(\mathbf{q}_{1}\right) V\left(\mathbf{q}_{2}\right) V\left(\mathbf{q}_{3}\right)\right\rangle=n_{i} v_{0}^{3} \delta\left(\mathbf{q}_{1}+\mathbf{q}_{2}+\mathbf{q}_{3}\right)$, we perform the impurity average 
and obtain

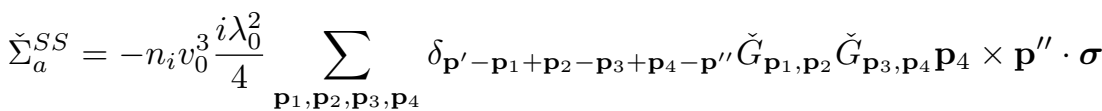

$$
\begin{aligned}
& \check{\Sigma}_{b}^{S S}=-n_{i} v_{0}^{3} \frac{i \lambda_{0}^{2}}{4} \sum_{\mathbf{p}_{1}, \mathbf{p}_{2}, \mathbf{p}_{3}, \mathbf{p}_{4}} \delta_{\mathbf{p}^{\prime}-\mathbf{p}_{1}+\mathbf{p}_{2}-\mathbf{p}_{3}+\mathbf{p}_{4}-\mathbf{p}^{\prime \prime}} \check{G}_{\mathbf{p}_{1}, \mathbf{p}_{2}} \mathbf{p}_{2} \times \mathbf{p}_{3} \cdot \boldsymbol{\sigma} \check{G}_{\mathbf{p}_{3}, \mathbf{p}_{4}} \\
& \check{\Sigma}_{c}^{S S}=-n_{i} v_{0}^{3} \frac{i \lambda_{0}^{2}}{4} \sum_{\mathbf{p}_{1}, \mathbf{p}_{2}, \mathbf{p}_{3}, \mathbf{p}_{4}} \delta_{\mathbf{p}^{\prime}-\mathbf{p}_{1}+\mathbf{p}_{2}-\mathbf{p}_{3}+\mathbf{p}_{4}-\mathbf{p}^{\prime \prime}} \mathbf{p}^{\prime} \times \mathbf{p}_{1} \cdot \boldsymbol{\sigma} \check{G}_{\mathbf{p}_{1}, \mathbf{p}_{2}} \check{G}_{\mathbf{p}_{3}, \mathbf{p}_{4}} .
\end{aligned}
$$

Let us introduce as before, momenta associated to center-of-mass and relative coordinates $\mathbf{p}=\left(\mathbf{p}^{\prime}+\mathbf{p}^{\prime \prime}\right) / 2, \mathbf{q}=\mathbf{p}^{\prime}-\mathbf{p}^{\prime \prime}, \widetilde{\mathbf{p}}_{a}=\left(\mathbf{p}_{1}+\mathbf{p}_{2}\right) / 2, \widetilde{\mathbf{q}}_{a}=$ $\mathbf{p}_{1}-\mathbf{p}_{2}, \widetilde{\mathbf{p}}_{b}=\left(\mathbf{p}_{3}+\mathbf{p}_{4}\right) / 2, \widetilde{\mathbf{q}}_{b}=\mathbf{p}_{3}-\mathbf{p}_{4}$ and we get after integrating over the momentum $\widetilde{\mathbf{q}}_{b}$

$$
\begin{aligned}
& \check{\Sigma}_{a}^{S S}=-n_{i} v_{0}^{3} \frac{i \lambda_{0}^{2}}{4} \sum_{\widetilde{\mathbf{p}}_{a}, \widetilde{\mathbf{p}}_{b}, \widetilde{\mathbf{q}}_{a}} \check{G}_{\widetilde{\mathbf{p}}_{a}, \widetilde{\mathbf{q}}_{a}} \check{G}_{\widetilde{\mathbf{p}}_{b}, \mathbf{q}-\widetilde{\mathbf{q}}_{a}}\left(\widetilde{\mathbf{p}}_{b}-\frac{\mathbf{q}}{2}+\frac{\widetilde{\mathbf{q}}_{a}}{2}\right) \times\left(\mathbf{p}-\frac{\mathbf{q}}{2}\right) \cdot \boldsymbol{\sigma} \\
& \check{\Sigma}_{b}^{S S}=-n_{i} v_{0}^{3} \frac{i \lambda_{0}^{2}}{4} \sum_{\widetilde{\mathbf{p}}_{a}, \widetilde{\mathbf{p}}_{b}, \widetilde{\mathbf{q}}_{a}} \check{G}_{\widetilde{\mathbf{p}}_{a}, \widetilde{\mathbf{q}}_{a}}\left(\widetilde{\mathbf{p}}_{a}-\frac{\widetilde{\mathbf{q}}_{a}}{2}\right) \times\left(\widetilde{\mathbf{p}}_{b}+\frac{\mathbf{q}}{2}-\frac{\widetilde{\mathbf{q}}_{a}}{2}\right) \cdot \boldsymbol{\sigma} \check{G}_{\widetilde{\mathbf{p}}_{b}, \mathbf{q}-\widetilde{\mathbf{q}}_{a}} \\
& \check{\Sigma}_{c}^{S S}=-n_{i} v_{0}^{3} \frac{i \lambda_{0}^{2}}{4} \sum_{\widetilde{\mathbf{p}}_{a}, \widetilde{\mathbf{p}}_{b}, \widetilde{\mathbf{q}}_{a}}\left(\mathbf{p}+\frac{\mathbf{q}}{2}\right) \times\left(\widetilde{\mathbf{p}}_{a}+\frac{\widetilde{\mathbf{q}}_{a}}{2}\right) \cdot \boldsymbol{\sigma} \check{G}_{\widetilde{\mathbf{p}}_{a}, \widetilde{\mathbf{q}}_{a}} \check{G}_{\widetilde{\mathbf{p}}_{b}, \mathbf{q}-\widetilde{\mathbf{q}}_{a}} \cdot
\end{aligned}
$$

We Fourier transform back with respect to the momentum $\mathbf{q}$ and neglect derivatives with respect to $\mathbf{r}$, i.e. we confine to lowest order in the gradient expansion. We then get

$$
\begin{aligned}
& \check{\Sigma}_{a}^{S S}=-n_{i} v_{0}^{3} \frac{i \lambda_{0}^{2}}{4} \sum_{\widetilde{\mathbf{p}}_{a}, \widetilde{\mathbf{p}}_{b}} \check{G}\left(\widetilde{\mathbf{p}}_{a}, \mathbf{r}\right) \check{G}\left(\widetilde{p}_{b}, \mathbf{r}\right) \widetilde{\mathbf{p}}_{b} \times p \cdot \boldsymbol{\sigma} \\
& \check{\Sigma}_{b}^{S S}=-n_{i} v_{0}^{3} \frac{i \lambda_{0}^{2}}{4} \sum_{\widetilde{p}_{a}, \widetilde{\mathbf{p}}_{b}} \check{G}\left(\widetilde{\mathbf{p}}_{a}, \mathbf{r}\right) \widetilde{\mathbf{p}}_{a} \times \widetilde{\mathbf{p}}_{b} \cdot \boldsymbol{\sigma} \check{G}\left(\widetilde{\mathbf{p}}_{b}, \mathbf{r}\right) \\
& \check{\Sigma}_{c}^{S S}=-n_{i} v_{0}^{3} \frac{i \lambda_{0}^{2}}{4} \sum_{\widetilde{\mathbf{p}}_{a}, \widetilde{\mathbf{p}}_{b}} \mathbf{p} \times \widetilde{\mathbf{p}}_{a} \cdot \boldsymbol{\sigma} \check{G}\left(\widetilde{\mathbf{p}}_{a}, \mathbf{r}\right) \check{G}\left(\widetilde{\mathbf{p}}_{b}, \mathbf{r}\right) .
\end{aligned}
$$

When Rashba SOC is present one has to consider the covariant self-energy, as done for the side jump and spin current swapping contribution. To leading order in the gradient expansion, this is done simply by replacing the Green function $\check{G}$ with its covariant expression $\check{\tilde{G}}$. Hence the self-energy 
responsible for the skew scattering reads

$$
\begin{aligned}
& \check{\tilde{\Sigma}}_{a}^{\mathrm{SS}}=-\mathrm{i} n_{i} v_{0}^{3} \frac{\lambda_{0}^{2}}{4} \sum_{\mathbf{p}_{a}, \mathbf{p}_{b}} \tilde{\tilde{G}}\left(\mathbf{p}_{a}, \mathbf{r}\right) \tilde{\tilde{G}}\left(\mathbf{p}_{b}, \mathbf{r}\right) \mathbf{p}_{b} \times \mathbf{p} \cdot \boldsymbol{\sigma} \\
& \check{\tilde{\Sigma}}_{b}^{\mathrm{SS}}=-\mathrm{i} n_{i} v_{0}^{3} \frac{\lambda_{0}^{2}}{4} \sum_{\mathbf{p}_{a}, \mathbf{p}_{b}} \tilde{\tilde{G}}\left(\mathbf{p}_{a}, \mathbf{r}\right) \mathbf{p}_{a} \times \mathbf{p}_{b} \cdot \boldsymbol{\sigma} \tilde{\tilde{G}}\left(\mathbf{p}_{b}, \mathbf{r}\right) \\
& \check{\tilde{\Sigma}}_{c}^{\mathrm{SS}}=-\mathrm{i} n_{i} v_{0}^{3} \frac{\lambda_{0}^{2}}{4} \sum_{\mathbf{p}_{a}, \mathbf{p}_{b}} \mathbf{p} \times \mathbf{p}_{a} \cdot \boldsymbol{\sigma} \tilde{\tilde{G}}\left(\mathbf{p}_{a}, \mathbf{r}\right) \tilde{\tilde{G}}\left(\mathbf{p}_{b}, \mathbf{r}\right) .
\end{aligned}
$$

Since we are considering the effect to first order in $\lambda_{0}^{2}$, the covariant Green functions entering Eqs.(124-126) are spin independent and isotropic in momentum space. As a result the retarded and advanced components of the above self-energies vanish, while the Keldysh component survives only for $\tilde{\Sigma}_{a}^{S S}$ and $\check{\tilde{\Sigma}}_{c}^{S S}$. Their joint contribution, after recalling that $\sum_{\mathbf{p}} \tilde{G}^{R}(\mathbf{p})=-\mathrm{i} \pi N_{0}$, leads to an extra term on the right hand side of the Boltzmann equation

$$
I^{S S}[f]=-2 \pi n_{i} v_{0}^{2}\left(v_{0} \pi N_{0}\right) \frac{\lambda_{0}^{2}}{4} \sum_{\mathbf{p}^{\prime}} \delta\left(\epsilon_{\mathbf{p}}-\epsilon_{\mathbf{p}^{\prime}}\right)\left\{\mathbf{p}^{\prime} \times \mathbf{p} \cdot \boldsymbol{\sigma}, f_{\mathbf{p}^{\prime}}\right\} .
$$

Finally by collecting the collision integrals Eq. (111) for spin current swapping, Eq. (116) for side jump and Eq. (127) for skew scattering, the Boltzmann equation Eq. (43) reads now

$$
\begin{aligned}
\tilde{\partial}_{t} f_{\mathbf{p}} & +\tilde{\nabla}_{\mathbf{r}} \cdot\left[\frac{\mathbf{p}}{m} f_{\mathbf{p}}+\frac{\lambda_{0}^{2}}{8 \tau}\left\langle\left\{\boldsymbol{\sigma} \times\left(\mathbf{p}^{\prime}-\mathbf{p}\right), f_{\mathbf{p}^{\prime}}\right\}\right\rangle\right] \\
& -\frac{e}{2}\left\{\left(\mathbf{E}+\frac{\mathbf{p}}{m} \times \mathbf{B} \cdot \nabla_{\mathbf{p}}\right), f_{\mathbf{p}}\right\} \\
& =-\frac{1}{\tau}\left(f_{\mathbf{p}}-\left\langle f_{\mathbf{p}^{\prime}}\right\rangle\right)-\left(\pi v_{0} N_{0}\right) \frac{\lambda_{0}^{2}}{4 \tau}\left\langle\left\{\mathbf{p}^{\prime} \times \mathbf{p} \cdot \boldsymbol{\sigma}, f_{\mathbf{p}^{\prime}}\right\}\right\rangle \\
& -i \frac{\lambda_{0}^{2}}{4 \tau}\left\langle\left[\boldsymbol{\sigma} \cdot \mathbf{p} \times \mathbf{p}^{\prime}, f_{\mathbf{p}^{\prime}}\right]\right\rangle \\
& +\frac{\lambda_{0}^{2}}{8 \tau}\left\langle\left(\left\{\tilde{\nabla}_{\mathbf{r}}\left(\boldsymbol{\sigma} \times \mathbf{p}^{\prime}\right), f_{\mathbf{p}^{\prime}}\right\}-\left\{\tilde{\nabla}_{\mathbf{r}}(\boldsymbol{\sigma} \times \mathbf{p}), f_{\mathbf{p}}\right\}\right)\right\rangle
\end{aligned}
$$

where, being the scattering elastic, $f_{\mathbf{p}}=f\left(\epsilon_{\mathbf{p}}, \hat{\mathbf{p}}\right)$ and $f_{\mathbf{p}^{\prime}}=f\left(\epsilon_{\mathbf{p}}, \hat{\mathbf{p}}^{\prime}\right)$ with $\langle\ldots\rangle$ indicating the integration over the directions of $\mathbf{p}^{\prime}$. Equation (128) is the Boltzmann equation valid to first order in the gradient expansion and up to first order in the extrinsic SOC $\lambda_{0}$. By setting to zero the Rashba SOC and any exchange field, the covariant derivatives only include the standard $\mathrm{U}(1)$ electromagnetic field. One can then derive the standard results for 
the spin Hall effect and spin current swapping

$$
\sigma_{s j}^{s H}=e \frac{\lambda_{0}^{2}}{4} n, \sigma_{s s}^{s H}=e \frac{\lambda_{0}^{2}}{4} n p_{F}^{2} \tau v_{0}, \kappa=\frac{\lambda_{0}^{2} p_{F}^{2}}{4},
$$

where $\sigma_{s j}^{s H}$ and $\sigma_{s s}^{s H}$ are the spin Hall conductivities ${ }^{2}$ for the side jump and skew scattering contribution and $\kappa$ is the spin current swapping coefficient ${ }^{17}$. When the Rashba SOC is present, the above equation allows the analysis of the interplay between the intrinsic and extrinsic SOC. However, it turns out that such interplay has some subtle aspects, which have led to the suggestion of an non analytical behavior for vanishing Rashba coupling $\alpha^{18-20}$. In the next section we will consider these aspects in detail and show that, indeed, there is no need to invoke a non-analyticity. Rather, one must take into account the fact that the extrinsic SOC introduces a further spin relaxation mechanism.

\section{The impurity-induced spin-orbit coupling: Elliott-Yafet spin relaxation}

The spin-orbit interaction with scattering centers, Eq. (91), gives rise also to spin-flip events leading to the Elliott-Yafet spin relaxation. Such a process is $\mathcal{O}\left(\lambda_{0}^{4}\right)$ and shown diagramatically in Fig. 4. Performing the impurity average and defining momenta as in Eq. (95), the Elliott-Yafet self-energy reads

$$
\begin{aligned}
\check{\Sigma}^{E Y}(\mathbf{p}, \mathbf{q})= & -\frac{\lambda_{0}^{4}}{16} n_{i} v_{0}^{2} \sum_{\tilde{\mathbf{p}}}[(\mathbf{p}+\mathbf{q} / 2) \times(\tilde{\mathbf{p}}+\mathbf{q} / 2)] \cdot \boldsymbol{\sigma} \check{G}_{\tilde{\mathbf{p}}, \mathbf{q}} \\
& \boldsymbol{\sigma} \cdot[(\tilde{\mathbf{p}}-\mathbf{q} / 2) \times(\mathbf{p}-\mathbf{q} / 2)] \\
& \approx \frac{\lambda_{0}^{4}}{16} n_{i} v_{0}^{2} \sum_{\tilde{\mathbf{p}}}(\mathbf{p} \times \tilde{\mathbf{p}}) \cdot \boldsymbol{\sigma} \check{G}_{\tilde{\mathbf{p}}, \mathbf{q}} \boldsymbol{\sigma} \cdot(\mathbf{p} \times \tilde{\mathbf{p}}) .
\end{aligned}
$$

In the last line subleading gradient terms $\mathcal{O}(\mathbf{q})$ have been neglected. Considering a $2 \mathrm{D}$ system, so that all momenta lie in the $x-y$ plane, and transforming back to the center-of-mass coordinate $\mathbf{r}$, one has (renaming $\tilde{\mathbf{p}} \rightarrow \mathbf{p}^{\prime}$ )

$$
\check{\tilde{\Sigma}}^{E Y}(\mathbf{p}, \mathbf{r})=\frac{\lambda_{0}^{4}}{16} n_{i} v_{0}^{2} \sum_{\mathbf{p}^{\prime}} \sigma^{z} \check{\tilde{G}}\left(\mathbf{p}^{\prime}, \mathbf{r}\right) \sigma^{z}\left(\mathbf{p} \times \mathbf{p}^{\prime}\right)_{z}^{2} .
$$

Notice that the Elliott-Yafet self-energy is already second order in the (extrinsic) SOC strength, so that its standard and covariant forms coincide up to $\mathcal{O}\left(\lambda_{0}^{4} \alpha\right)$. Such higher-order corrections are not needed here, and therefore covariant quantities were directly introduced in Eq. (131). The 


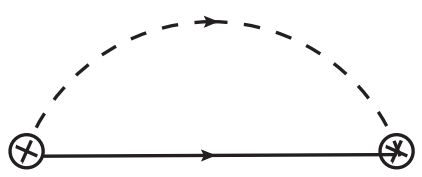

Fig. 4. Self-energy diagram in second order in the spin-orbit impurity potential contributing to the Elliott-Yafet spin relaxation.

expression (131) yields the following extra collision term on the right hand side of the Boltzmann equation Eq. (128):

$$
I^{E Y}[f]=-\frac{\lambda_{0}^{4}}{16} 2 \pi n_{i} v_{0}^{2} \sum_{\mathbf{p}^{\prime}} \delta\left(\epsilon_{\mathbf{p}}-\epsilon_{\mathbf{p}^{\prime}}\right)\left[f_{\mathbf{p}}-\sigma^{z} f_{\mathbf{p}^{\prime}} \sigma^{z}\right]\left(\mathbf{p} \times \mathbf{p}^{\prime}\right)_{z}^{2} .
$$

The two $\sigma^{z}$ Pauli matrices flip the in-plane spin components of the distribution function, $\sigma^{z}\left(f_{\mathbf{p}^{\prime}}^{x, y} \sigma^{x, y}\right) \sigma^{z}=-f_{\mathbf{p}^{\prime}}^{x, y} \sigma^{x, y}$, while leaving the out-of-plane component $f_{\mathbf{p}^{\prime}}^{z} \sigma^{z}$ unchanged. This is a signature that spin-orbit interaction with the random $2 \mathrm{D}$ impurity potential conserves the $z$-spin component, while relaxing only the in-plane spins - a result valid strictly in $2 \mathrm{D} .{ }^{\mathrm{d}} \mathrm{In}$ order to see this more clearly we follow the procedure of Sec. 3, and compute the modification to the spin equation of motion (62) according to

$$
\frac{1}{2} \operatorname{Tr}\left[\sigma^{a} \sum_{\mathbf{p}} \delta \tilde{I}^{E Y}[f]\right]=-\hat{\Gamma}_{E Y}^{a b} s^{b},
$$

where $\hat{\Gamma}_{E Y}$ is the Elliott-Yafet spin relaxation matrix. Explicitly

$$
\begin{aligned}
\frac{1}{2} \operatorname{Tr}\left[\sigma^{x, y} \sum_{\mathbf{p}} \delta \tilde{I}^{E Y}[f]\right] & =-\frac{\lambda_{0}^{4}}{16} 2 \pi n_{i} v_{0}^{2} \sum_{\mathbf{p}, \mathbf{p}^{\prime}} \delta\left(\epsilon_{\mathbf{p}}-\epsilon_{\mathbf{p}^{\prime}}\right)\left[f_{\mathbf{p}}^{x, y}+f_{\mathbf{p}^{\prime}}^{x, y}\right]\left(\mathbf{p} \times \mathbf{p}^{\prime}\right)_{z}^{2} \\
& \approx-\frac{1}{\tau}\left(\frac{\lambda_{0} p_{F}}{2}\right)^{4} s^{x, y}
\end{aligned}
$$

and

$$
\begin{aligned}
\frac{1}{2} \operatorname{Tr}\left[\sigma^{z} \sum_{\mathbf{p}} \delta \tilde{I}^{E Y}[f]\right] & =-\frac{\lambda_{0}^{4}}{16} 2 \pi n_{i} v_{0}^{2} \sum_{\mathbf{p}, \mathbf{p}^{\prime}} \delta\left(\epsilon_{\mathbf{p}}-\epsilon_{\mathbf{p}^{\prime}}\right)\left[f_{\mathbf{p}}^{z}-f_{\mathbf{p}^{\prime}}^{z}\right]\left(\mathbf{p} \times \mathbf{p}^{\prime}\right)_{z}^{2} \\
& =0
\end{aligned}
$$

\footnotetext{
${ }^{\mathrm{d}}$ One may consider in certain circumstances a more general model including also random Rashba SOC ${ }^{21}$.
} 
implying

$$
\hat{\Gamma}_{E Y}=\frac{1}{\tau_{E Y}}\left(\begin{array}{lll}
1 & 0 & 0 \\
0 & 1 & 0 \\
0 & 0 & 0
\end{array}\right), \quad \frac{1}{\tau_{E Y}}=\frac{1}{\tau}\left(\frac{\lambda_{0} p_{F}}{2}\right)^{4} .
$$

In order to obtain Eq. (134) we employed the diffusive expansion (46) and set

$$
\left\langle f_{\mathbf{p}}\right\rangle \approx \frac{1}{N_{0}} \delta\left(\epsilon_{\mathbf{p}}-\epsilon_{F}\right) s^{a},
$$

which is appropriate for the present paramagnetic case. On the other hand Eq. (135) is obtained at once by noticing that $\left(\mathbf{p} \times \mathbf{p}^{\prime}\right)_{z}^{2}$ is even under exchange $\mathbf{p} \leftrightarrow \mathbf{p}^{\prime}$.

Elliott-Yafet relaxation, though typically weak, plays a crucial role in the appropriate description of the spin Hall effect in 2DEGs. Indeed, the first efforts to combine intrinsic and extrinsic contributions to the spin Hall effect lead to a puzzling non-analytical behaviour ${ }^{18-20}$ : the spin Hall conductivity for a purely extrinsic sample, with $\alpha=0$, differed from the value obtained by considering a system with both mechanisms present, where however $\alpha \rightarrow 0$. This unphysical behaviour is cured by Elliott-Yafet processes ${ }^{22}$. To be definite, we follow Ref. 2 and consider the coupled dynamics of $J_{y}^{z}$ and $s^{y}$ in the presence of a $x$-pointing electric field (cf. Eq. (69) and Eq. (72)). The spin current is

$$
J_{y}^{z}=2 m \alpha D s^{y}+\left(\sigma_{i n t}^{s H}+\sigma_{e x t}^{s H}\right) E_{x},
$$

with $\sigma_{e x t}^{s H}=\sigma_{s j}^{s H}+\sigma_{s s}^{s H}$, while from Eqs. (62) and (136) one has for $s^{y}$

$$
\partial_{t} s^{y}=-\frac{1}{\tau_{E Y}}-2 m \alpha J_{y}^{z} .
$$

Solving Eqs. (138)-(139) yields ${ }^{2}$

$$
\begin{aligned}
J_{y}^{z} & =\frac{1}{1+\tau_{E Y} / \tau_{D P}}\left(\sigma_{i n t}^{s H}+\sigma_{e x t}^{s H}\right) E_{x} \\
s^{y} & =-\frac{2 m \alpha \tau_{E Y}}{1+\tau_{E Y} / \tau_{D P}}\left(\sigma_{i n t}^{s H}+\sigma_{e x t}^{s H}\right) E_{x},
\end{aligned}
$$

which are respectively the generalisation of the spin Hall and inverse spin galvanic/Edelstein effects in the presence of intrinsic (Rashba) and extrinsic spin-orbit coupling. These expressions are analytical and reduce to the known results for either $\alpha \rightarrow 0$ or $\lambda_{0}^{2} \rightarrow 0$. Physically, they show that the behaviour of both effects is determined by the ratio between DyakonovPerel and Elliott-Yafet spin relaxation. 


\section{Conclusions}

We have employed the Keldysh formalism - in its semiclassical limit - to describe the spin-charge coupled dynamics in a 2DEG with both intrinsic (Rashba) and extrinsic sources of spin-orbit interaction. Such dynamics are rich and typically rather intricated, but we have seen that rewriting spin-orbit coupling in terms of non-Abelian gauge fields leads to a compact and physically transparent set of equations. In particular the latter, obtained from the equations of motion of the locally $S U(2)$-covariant Keldysh Green's function $\tilde{\widetilde{G}}(1,2)$, show that:

- Spin and charge are coupled via the $S U(2)$ field tensor ("spinelectric" and "spin-magnetic" fields);

- The spin obeys an $S U(2)$-covariant continuity equation, appropriately modified when extrinsic spin-orbit is present; this corrects both the definition of the spin current and the collision integral, but preserves their covariance properties;

- The side-jump mechanism is naturally seen as a modification of the velocity operator arising from the $\mathbf{q} \neq 0$ corrections to the Born self-energy;

- Skew scattering and spin current swapping are interference processes proportional to $\operatorname{Re}\left(A B^{*}\right)$ and $\operatorname{Im}\left(A B^{*}\right)$, respectively, where $A$ and $B$ are the scattering amplitudes defined in Eq. (92). Skew scattering arises beyond the Born approximation, which is instead enough to have the spin current swapping. Both processes, in contrast with side jump, are due to $\mathbf{q}=0$ self-energy terms;

- Elliott-Yafet spin relaxation introduces a typically small though crucial energy scale, which is necessary to cure unphysical nonanalytical behaviours of various physical quantities.

The Keldysh non-Abelian approach we have outlined focusing on the paradigmatic Rashba 2DEG can actually be (and indeed has been) employed in a wide range of systems, and offers certain further advantages. Let us briefly mention a few.

- The lack of spin conservation in the presence of spin-orbit coupling may lead to ambiguous definitions of, e.g. spin currents. This was extensively debated from early on ${ }^{23}$ and posed problems concerning Onsager reciprocity. ${ }^{24}$ Such problems were solved via the non-Abelian formulation, ${ }^{25,26}$ by construction devoid of any ambiguity. ${ }^{27}$ 
- The kinetic equations can include any linear-in-momentum spinorbit field, e.g. à la Rashba-Dresselhaus. Furthermore, (pseudo)spin-orbit coupling in $N$-band models can be written in terms of $S U(N)$ gauge fields, and formally handled exactly as we did in the single band, $2 \times 2$ case.

- The formalism can describe the dynamics of exotic systems, such as cold atoms in artificial gauge fields. ${ }^{28}$

- Non-homogeneous and/or dynamical spin-orbit coupling, e.g. gatecontrolled $^{1,29,30}$ or due to thermal vibrations ${ }^{31}$, has numerous practical and theoretical implications, and is by construction included in the non-Abelian approach. In a similar way, the latter can deal with the spin and charge dynamics induced by time-dependent magnetic textures. ${ }^{32}$

\section{References}

1. C. Gorini, P. Schwab, R. Raimondi and A. L. Shelankov, Non-Abelian gauge fields in the gradient expansion: Generalized Boltzmann and Eilenberger equations, Phys. Rev. B 82, p. 195316 (Nov 2010).

2. R. Raimondi, P. Schwab, C. Gorini and G. Vignale, Spin-orbit interaction in a two-dimensional electron gas: a $\mathrm{SU}(2)$ formulation, Ann. Phys. 524, p. 153 (2012).

3. G. Tatara, Theory of electron transport and magnetization dynamics in metallic ferromagnets, in this lecture series (2017).

4. J. Rammer, Quantum Field Theory of Nonequilibrium States (Cambridge University Press, Cambridge, 2007).

5. A. A. Abrikosov, L. P. Gorkov and I. E. Dzyaloshinski, Methods of Quantum Field Theory in Statistical Physics (Dover Publications, 1975).

6. Y. A. Bychkov and E. I. Rashba, Oscillatory effects and the magnetic susceptibility of carriers in inversion layers, J. Phys. C 17, p. 6039 (1984).

7. V. M. Edelstein,, Solid State Commun. 73, p. 233 (1990).

8. A. G. Aronov and Y. B. Lyanda-Geller, JETP Lett. 50, p. 431 (1989).

9. S. D. Ganichev, E. L. Ivchenko, V. V. Belkov, S. A. Tarasenko, M. Sollinger, D. Weiss, W. Wegscheider and W. Prettl, Spin-galvanic effect, Nature 417, p. 153 (2002).

10. S. D. Ganichev, M. Trushin and J. Schliemann, Spin polarisation by current, ArXiv e-prints (June 2016). 
11. R. Raimondi and P. Schwab, , Phys. Rev. B 71, p. 033311 (2005).

12. S. Tölle, C. Gorini and U. Eckern, Room-temperature spin thermoelectrics in metallic films, Phys. Rev. B 90, p. 235117 (Dec 2014).

13. H.-A. Engel, B. I. Halperin and E. Rashba, Theory of Spin Hall Conductivity in n-Doped GaAs, Phys. Rev. Lett. 95, p. 166605 (2005).

14. W.-K. Tse and S. Das Sarma, Spin hall effect in doped semiconductor structures (2006).

15. M. B. Lifshits and M. I. Dyakonov, Swapping spin currents: Interchanging spin and flow directions, Phys. Rev. Lett. 103, p. 186601 (Oct 2009).

16. L. Berger, Side-jump mechanism for the hall effect of ferromagnets, Phys. Rev. B 2, 4559 (Dec 1970).

17. K. Shen, R. Raimondi and G. Vignale, Theory of coupled spincharge transport due to spin-orbit interaction in inhomogeneous twodimensional electron liquids, Phys. Rev. B 90, p. 245302 (Dec 2014).

18. W.-K. Tse and S. Das Sarma, , Phys. Rev. B 74, p. 245309 (2006).

19. E. M. Hankiewicz and G. Vignale, Phase Diagram of the Spin Hall Effect, Phys. Rev. Lett. 100, p. 026602 (2008).

20. J. L. Cheng and M. W. Wu, Kinetic investigation of the extrinsic spin Hall effect induced by skew scattering, Journal of Physics: Condensed Matter 20, p. 085209 (2008).

21. V. K. Dugaev, E. Y. Sherman, V. I. Ivanov and J. Barnaś, Spin relaxation and combined resonance in two-dimensional electron systems with spin-orbit disorder, Phys. Rev. B 80, p. 081301 (Aug 2009).

22. R. Raimondi and P. Schwab, Tuning the Spin Hall Effect in a TwoDimensional Electron Gas, Europhys. Lett. 87, p. 37008 (2009).

23. E. I. Rashba, Spin currents in thermodynamic equilibrium: The challenge of discerning transport currents, Phys. Rev. B 68, p. 241315(R) (2003).

24. L. Y. Wang, A. G. Mal'shukov and C. S. Chu, Nonuniversality of the intrinsic inverse spin-Hall effect in diffusive systems, Phys. Rev. B 85, p. 165201 (2012).

25. C. Gorini, R. Raimondi and P. Schwab, Onsager Relations in a TwoDimensional Electron Gas with Spin-Orbit Coupling, Phys. Rev. Lett. 109, p. 246604 (2012).

26. K. Shen, G. Vignale and R. Raimondi, Microscopic Theory of the Inverse Edelstein Effect, Phys. Rev. Lett. 112, p. 096601 (Mar 2014).

27. I. V. Tokatly, Equilibrium Spin Currents: Non-Abelian Gauge Invariance and Color Diamagnetism in Condensed Matter, Phys. Rev. Lett. 
101, p. 106601 (2008).

28. I. V. Tokatly and E. Y. Sherman, Spin evolution of cold atomic gases in $S U(2) \otimes U(1)$ fields, Phys. Rev. A 93, p. 063635 (2016).

29. P. W. Brouwer, J. N. H. J. Cremers and B. I. Halperin, Weak localization and conductance fluctuations of a chaotic quantum dot with tunable spin-orbit coupling, Phys. Rev. B 65, p. 081302(R) (2002).

30. A. G. Mal'shukov, C. S. Tang, C. S. Chu and K. A. Chao, Spin-current generation and detection in the presence of an ac gate, Phys. Rev. B 68, p. 233307 (2003).

31. C. Gorini, U. Eckern and R. Raimondi, Spin Hall Effects Due to Phonon Skew Scattering, Phys. Rev. Lett. 115, p. 076602 (2015).

32. Y. Tserkovnyak and M. Mecklenburg, Electron transport driven by nonequilibrium magnetic textures, Phys. Rev. B 77, p. 134407 (2008). 